Article

\title{
Convergence Analysis of Self-Adaptive Inertial Extra-Gradient Method for Solving a Family of Pseudomonotone Equilibrium Problems with Application
}

\author{
Thanatporn Bantaojai ${ }^{1}$, Nuttapol Pakkaranang ${ }^{2}{ }^{(}$, Habib ur Rehman ${ }^{2}{ }^{(1)}$, \\ Poom Kumam $2,3,4, * \mathbb{D}$ and Wiyada Kumam ${ }^{5, * \mathbb{D}}$ \\ 1 Mathematics English Program, Faculty of Education, Valaya Alongkorn Rajabhat University under the \\ Royal Patronage, Pathumthani 13180, Thailand; thanatporn.ban@vru.ac.th \\ 2 KMUTTFixed Point Research Laboratory, KMUTT-Fixed Point Theory and Applications Research Group, \\ SCL 802 Fixed Point Laboratory, Department of Mathematics, Faculty of Science, King Mongkut's University \\ of Technology Thonburi (KMUTT), 126 Pracha-Uthit Road, Bang Mod, Thrung Khru, \\ Bangkok 10140, Thailand; nuttapol.pakka@gmail.com (N.P.); hrehman.hed@gmail.com (H.u.R.) \\ 3 Center of Excellence in Theoretical and Computational Science (TaCS-CoE), Science Laboratory Building, \\ King Mongkut's University of Technology Thonburi (KMUTT), 126 Pracha-Uthit Road, Bang Mod, \\ Thrung Khru, Bangkok 10140, Thailand \\ 4 Department of Medical Research, China Medical University Hospital, China Medical University, \\ Taichung 40402, Taiwan \\ 5 Program in Applied Statistics, Department of Mathematics and Computer Science, Faculty of Science and \\ Technology, Rajamangala University of Technology Thanyaburi, Thanyaburi, Pathumthani 12110, Thailand \\ * Correspondence: poom.kum@kmutt.ac.th (P.K.); wiyada.kum@rmutt.ac.th (W.K.)
}

Received: 24 June 2020; Accepted: 6 August 2020; Published: 10 August 2020

check for updates

\begin{abstract}
In this article, we propose a new modified extragradient-like method to solve pseudomonotone equilibrium problems in real Hilbert space with a Lipschitz-type condition on a bifunction. This method uses a variable stepsize formula that is updated at each iteration based on the previous iterations. The advantage of the method is that it operates without prior knowledge of Lipschitz-type constants and any line search method. The weak convergence of the method is established by taking mild conditions on a bifunction. In the context of an application, fixed-point theorems involving strict pseudo-contraction and results for pseudomonotone variational inequalities are considered. Many numerical results have been reported to explain the numerical behavior of the proposed method.
\end{abstract}

Keywords: pseudomonotone bifunction; convex optimization; equilibrium problems; variational inequality problems; weak convergence

\section{Introduction}

Let $\mathcal{C}$ be a nonempty, closed and convex subset of a real Hilbert space $\mathcal{H}$ and $\mathcal{R}, \mathcal{N}$ be the sets of real numbers and natural numbers, respectively. Assume that $f$ is a bifunction $f: \mathcal{H} \times \mathcal{H} \rightarrow \mathcal{R}$ and $E P(f, \mathcal{C})$ denotes the solution set of an equilibrium problem over the set $\mathcal{C}$. Now, consider the following definitions of a bifunction monotonicity (see [1,2] for more details). A function $f: \mathcal{H} \times \mathcal{H} \rightarrow \mathcal{R}$ on $\mathcal{C}$ for $\gamma>0$ is said to be:

(1) $\gamma$-strongly monotone if

$$
f\left(z_{1}, z_{2}\right)+f\left(z_{2}, z_{1}\right) \leq-\gamma\left\|z_{1}-z_{2}\right\|^{2}, \forall z_{1}, z_{2} \in \mathcal{C}
$$


(2) monotone if

$$
f\left(z_{1}, z_{2}\right)+f\left(z_{2}, z_{1}\right) \leq 0, \forall z_{1}, z_{2} \in \mathcal{C} ;
$$

(3) $\gamma$-strongly pseudomonotone if

$$
f\left(z_{1}, z_{2}\right) \geq 0 \Longrightarrow f\left(z_{2}, z_{1}\right) \leq-\gamma\left\|z_{1}-z_{2}\right\|^{2}, \forall z_{1}, z_{2} \in \mathcal{C} ;
$$

(4) pseudomonotone if

$$
f\left(z_{1}, z_{2}\right) \geq 0 \Longrightarrow f\left(z_{2}, z_{1}\right) \leq 0, \forall z_{1}, z_{2} \in \mathcal{C}
$$

It is clear from the definitions mentioned above that they have the following consequences:

$$
(1) \Longrightarrow(2) \Longrightarrow(4) \text { and }(1) \Longrightarrow(3) \Longrightarrow(4) \text {. }
$$

In general, the converses are not true. A bifunction $f: \mathcal{H} \times \mathcal{H} \rightarrow \mathcal{R}$ is said to be Lipschitz-type continuous on $\mathcal{C}$ if there exist two positive constants $c_{1}, c_{2}$ such that

$$
f\left(z_{1}, z_{3}\right) \leq f\left(z_{1}, z_{2}\right)+f\left(z_{2}, z_{3}\right)+c_{1}\left\|z_{1}-z_{2}\right\|^{2}+c_{2}\left\|z_{2}-z_{3}\right\|^{2}, \forall z_{1}, z_{2}, z_{3} \in \mathcal{C} .
$$

Let $\mathcal{C}$ be a nonempty closed convex subset of $\mathcal{H}$ and $f: \mathcal{H} \times \mathcal{H} \rightarrow \mathcal{R}$ be a bifunction with $f\left(z_{1}, z_{1}\right)=0$, for all $z_{1} \in \mathcal{C}$. An equilibrium problem $[1,3]$ for $f$ on the $\operatorname{set} \mathcal{C}$ is to

$$
\text { find } u^{*} \in \mathcal{C} \text { such that } f\left(u^{*}, z_{1}\right) \geq 0, \forall z_{1} \in \mathcal{C} \text {. }
$$

An equilibrium problem (1) had many mathematical problems as a particular case, i.e., the variational inequality problems (VIP), optimization problems, fixed point problems, complementarity problems, the Nash equilibrium of non-cooperative games, saddle point problems and the vector optimization problem (for details see [1,4,5]). The equilibrium problem is also known as the famous Ky Fan inequality [3]. However, the particular format of an equilibrium problem (1) was initiated by Muu and Oettli [6] in 1992 and further investigation on its theoretical properties were provided by Blum and Oettli [1]. The construction of new iterative schemes and the modification of existing methods, as well as the study their convergence analysis, constitute an important research direction in equilibrium problem theory. Several methods have been developed in the past few years to approximate the solution of an equilibrium problem in finite and infinite dimensional real Hilbert spaces, i.e., extragradient methods [7-16], subgradient methods [17-22], inertial methods [23-25] and methods for particular classes of equilibrium problems [26-35].

In particular, a proximal method [36] was used to solve equilibrium problems based on solving minimization problems. This approach was also known as the two-step extragradient-like method in [7] due to the early contribution of the Korpelevich [37] extragradient method to solve the saddle point problems. More precisely, Tran et al. introduced a method in [7], and an iterative sequence $\left\{u_{n}\right\}$ was generated as follows:

$$
\left\{\begin{array}{l}
u_{0} \in \mathcal{C}, \\
v_{n}=\arg \min \left\{\lambda f\left(u_{n}, v\right)+\frac{1}{2}\left\|u_{n}-v\right\|^{2}: v \in \mathcal{C}\right\}, \\
u_{n+1}=\arg \min \left\{\lambda f\left(v_{n}, v\right)+\frac{1}{2}\left\|u_{n}-v\right\|^{2}: v \in \mathcal{C}\right\},
\end{array}\right.
$$

where $0<\lambda<\min \left\{\frac{1}{2 c_{1}}, \frac{1}{2 c_{2}}\right\}$. The iterative sequence generated from the above-mentioned method provides a weak convergent iterative sequence and in order to operate it, prior information regarding the Lipschitz-type constants is required. These Lipschitz-type constants are mostly unknown or hard to compute. To overcome this situation, Hieu et al. [14] introduced an extension of the method in [38] 
for solving the equilibrium problem as follows: Let $[t]_{+}:=\max \{t, 0\}$ and choose $u_{0} \in \mathcal{C}, \mu \in(0,1)$ with $\lambda_{0}>0$ such that

$$
\left\{\begin{array}{l}
v_{n}=\arg \min \left\{\lambda_{n} f\left(u_{n}, v\right)+\frac{1}{2}\left\|u_{n}-v\right\|^{2}: v \in \mathcal{C}\right\}, \\
u_{n+1}=\arg \min \left\{\lambda_{n} f\left(v_{n}, v\right)+\frac{1}{2}\left\|u_{n}-v\right\|^{2}: v \in \mathcal{C}\right\}
\end{array}\right.
$$

where the stepsize sequence $\left\{\lambda_{n}\right\}$ is updated in the following way:

$$
\lambda_{n+1}=\min \left\{\lambda_{n}, \frac{\mu\left(\left\|u_{n}-v_{n}\right\|^{2}+\left\|u_{n+1}-v_{n}\right\|^{2}\right)}{2\left[f\left(u_{n}, u_{n+1}\right)-f\left(u_{n}, v_{n}\right)-f\left(v_{n}, u_{n+1}\right)\right]_{+}}\right\} .
$$

Recently, Vinh and Muu proposed an inertial iterative algorithm in [39] to solve a pseudomonotone equilibrium problem. Their main contribution is the availability of an inertial effect in the algorithm that is used to improve the convergence rate of the iterative sequence. The iterative sequence $\left\{u_{n}\right\}$ has been generated in the following manner:

(i) Choose $u_{-1}, u_{0} \in \mathcal{C}, \vartheta \in[0,1), 0<\lambda<\min \left\{\frac{1}{2 c_{1}}, \frac{1}{2 c_{2}}\right\}$ while a sequence $\left\{\epsilon_{n}\right\} \subset[0,+\infty)$ is satisfying the following condition:

$$
\sum_{n=0}^{+\infty} \epsilon_{n}<+\infty
$$

(ii) Choose $\vartheta_{n}$ such that $0 \leq \vartheta_{n} \leq \bar{\vartheta}_{n}$ where

$$
\bar{\vartheta}_{n}= \begin{cases}\min \left\{\vartheta, \frac{\epsilon_{n}}{\left\|u_{n}-u_{n-1}\right\|}\right\} & \text { if } u_{n} \neq u_{n-1} \\ \vartheta & \text { otherwise. }\end{cases}
$$

(iii) Determine

$$
\left\{\begin{array}{l}
\eta_{n}=u_{n}+\vartheta_{n}\left(u_{n}-u_{n-1}\right) \\
v_{n}=\arg \min \left\{\lambda f\left(\eta_{n}, v\right)+\frac{1}{2}\left\|\eta_{n}-v\right\|^{2}: v \in \mathcal{C}\right\} \\
u_{n+1}=\arg \min \left\{\lambda f\left(v_{n}, v\right)+\frac{1}{2}\left\|\eta_{n}-v\right\|^{2}: v \in \mathcal{C}\right\}
\end{array}\right.
$$

This article focuses on projection methods that are well-known and easy to execute due to their efficient and straightforward mathematical computation. Motivated by the works of $[14,40]$, we formulate an inertial explicit subgradient extragradient algorithm to solve the pseudomonotone equilibrium problem. The proposed algorithm can be seen as the modification of the methods that appear in $[7,14,39]$. Under certain mild conditions, a weak convergence result has been proven to correspond to the iterative sequence of the algorithm. Moreover, experimental studies have shown that the proposed method tends to be more efficient compared to the existing method [39].

The remainder of this paper is arranged as follows: Section 2 contains some definitions and basic results used in the paper. Section 3 contains our main algorithm and proves its convergence. Sections 4 and 5 incorporate the implementation of our results. Section 6 carries out the numerical results that demonstrates the computational effectiveness of our proposed algorithm.

\section{Background}

Let $h: \mathcal{C} \rightarrow \mathcal{R}$ be a convex function on a nonempty, closed and convex subset $\mathcal{C}$ of a real Hilbert space $\mathcal{H}$, and the subdifferential of a function $h$ at $z_{1} \in \mathcal{C}$ is defined as:

$$
\partial h\left(z_{1}\right)=\left\{z_{3} \in \mathcal{H}: h\left(z_{2}\right)-h\left(z_{1}\right) \geq\left\langle z_{3}, z_{2}-z_{1}\right\rangle, \forall z_{2} \in \mathcal{C}\right\} .
$$


Let $\mathcal{C}$ be a nonempty, closed and convex subset of a real Hilbert space $\mathcal{H}$ and a normal cone of $\mathcal{C}$ at $z_{1} \in \mathcal{C}$ is defined by:

$$
N_{\mathcal{C}}\left(z_{1}\right)=\left\{z_{3} \in \mathcal{H}:\left\langle z_{3}, z_{2}-z_{1}\right\rangle \leq 0, \forall z_{2} \in \mathcal{C}\right\} .
$$

The metric projection $P_{\mathcal{C}}\left(z_{1}\right)$ for $z_{1} \in \mathcal{H}$ onto a closed and convex subset $\mathcal{C}$ of $\mathcal{H}$ is defined by:

$$
P_{\mathcal{C}}\left(z_{1}\right)=\arg \min \left\{\left\|z_{2}-z_{1}\right\|: z_{2} \in \mathcal{C}\right\} .
$$

Lemma 1 ([41]). Let $\mathcal{C}$ be a nonempty, closed and convex subset of a real Hilbert space $\mathcal{H}$ and $P_{\mathcal{C}}: \mathcal{H} \rightarrow \mathcal{C}$ be a metric projection from $\mathcal{H}$ onto $\mathcal{C}$.

(i) Let $z_{1} \in \mathcal{C}$ and $z_{2} \in \mathcal{H}$; we have

$$
\left\|z_{1}-P_{\mathcal{C}}\left(z_{2}\right)\right\|^{2}+\left\|P_{\mathcal{C}}\left(z_{2}\right)-z_{2}\right\|^{2} \leq\left\|z_{1}-z_{2}\right\|^{2} .
$$

(ii) $z_{3}=P_{\mathcal{C}}\left(z_{1}\right)$ if and only if

$$
\left\langle z_{1}-z_{3}, z_{2}-z_{3}\right\rangle \leq 0, \forall z_{2} \in \mathcal{C}
$$

(iii) For $z_{2} \in \mathcal{C}$ and $z_{1} \in \mathcal{H}$

$$
\left\|z_{1}-P_{\mathcal{C}}\left(z_{1}\right)\right\| \leq\left\|z_{1}-z_{2}\right\|
$$

Lemma 2 ([42]). Let $h: \mathcal{C} \rightarrow \mathcal{R}$ be a convex, subdifferentiable and lower semicontinuous function on $\mathcal{C}$, where $\mathcal{C}$ is a nonempty, convex and closed subset of a real Hilbert space $\mathcal{H}$. Then, an element $z_{1} \in \mathcal{C}$ is a minimizer of a function $h$ if and only if $0 \in \partial h\left(z_{1}\right)+N_{\mathcal{C}}\left(z_{1}\right)$, where $\partial h\left(z_{1}\right)$ and $N_{\mathcal{C}}\left(z_{1}\right)$ represent the subdifferential of $h$ at $z_{1} \in \mathcal{C}$ and normal cone of $\mathcal{C}$ at $z_{1}$, respectively.

Lemma 3 ([43]). Let $\left\{u_{n}\right\}$ be a sequence in $\mathcal{H}$ and $\mathcal{C} \subset \mathcal{H}$ such that the following conditions hold:

(i) For each $u \in \mathcal{C}$, the $\lim _{n \rightarrow \infty}\left\|u_{n}-u\right\|$ exists;

(ii) Each sequentially weak cluster limit point of the sequence $\left\{u_{n}\right\}$ belongs to $\mathcal{C}$.

Then, the sequence $\left\{u_{n}\right\}$ weakly converges to some element in $\mathcal{C}$.

Lemma 4. [44] Let $\left\{q_{n}\right\}$ and $\left\{p_{n}\right\}$ be sequences of non-negative real numbers satisfying $q_{n+1} \leq q_{n}+p_{n}$, for each $n \in \mathcal{N}$. If $\sum p_{n}<\infty$, then $\lim _{n \rightarrow \infty} q_{n}$ exists.

Assume that a bifunction $f$ satisfies the following conditions:

(f1) $\quad f\left(z_{2}, z_{2}\right)=0$, for all $z_{2} \in \mathcal{C}$ and $f$ is pseudomonotone on $\mathcal{C}$;

(f2) $f$ satisfies the Lipschitz-type condition on $\mathcal{H}$ through $c_{1}>0$ and $c_{2}>0$;

(f3) $\quad \limsup _{n \rightarrow \infty} f\left(z_{n}, v\right) \leq f\left(z^{*}, v\right)$ for every $v \in \mathcal{C}$ and $\left\{z_{n}\right\} \subset \mathcal{C}$ satisfying $z_{n} \rightarrow z^{*}$;

(f4) $\quad f\left(z_{1},.\right)$ needs to be convex and subdifferentiable on $\mathcal{H}$ for each $z_{1} \in \mathcal{H}$. 


\section{Convergence Analysis for an Algorithm}

We provide a method consisting of two strongly convex minimization problems through an inertial factor and an explicit stepsize formula, which are being used to improve the convergence rate of the iterative sequence and to make the method independent of the Lipschitz constants. The detailed method is provided below Algorithm 1:

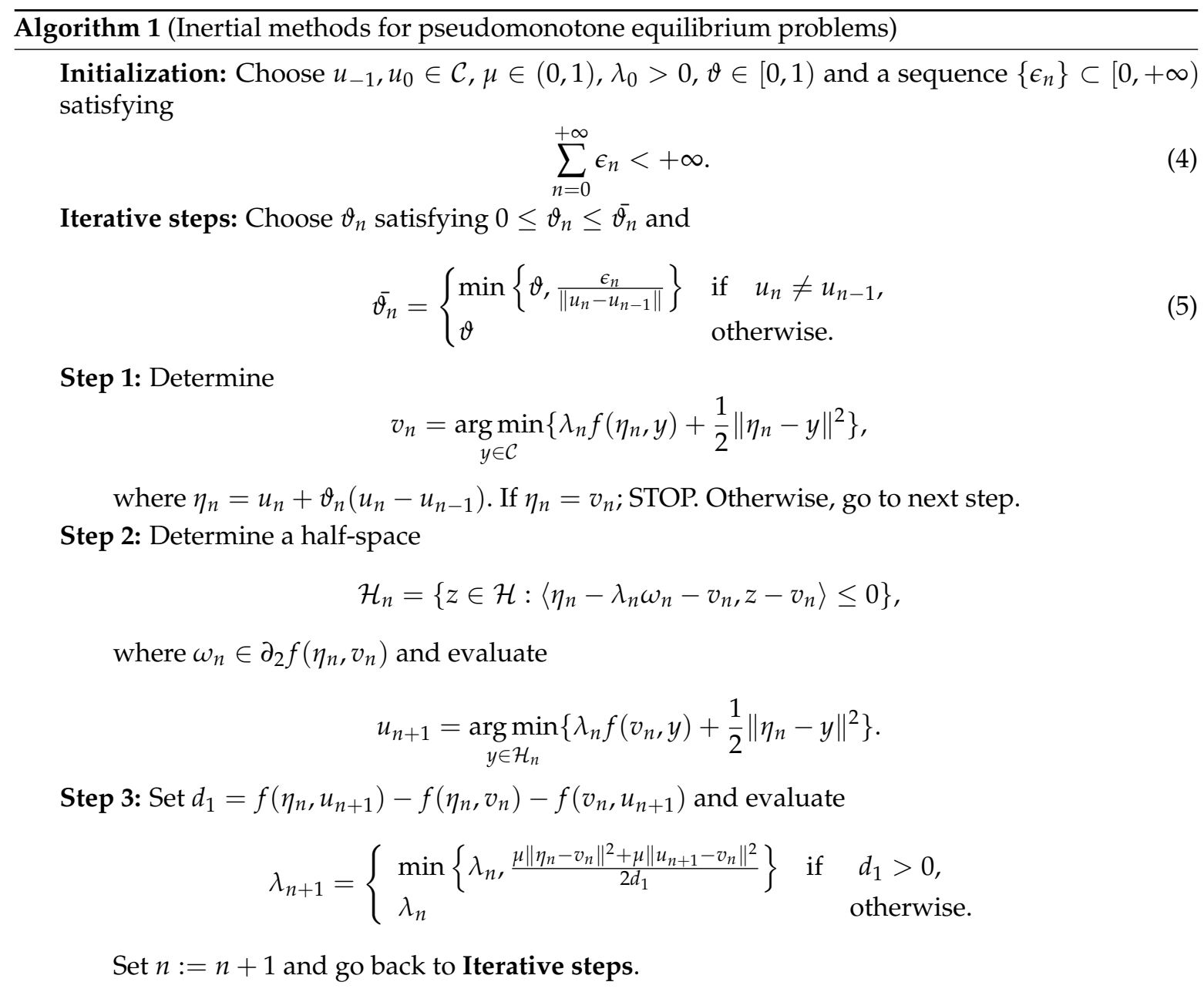

Lemma 5. The sequence $\left\{\lambda_{n}\right\}$ is decreasing monotonically with a lower bound $\min \left\{\frac{\mu}{2 \max \left\{c_{1}, c_{2}\right\}}, \lambda_{0}\right\}$ and converges to $\lambda>0$.

Proof. From the definition of $\left\{\lambda_{n}\right\}$, we see that this sequence is monotone and non-increasing. It is given that $f$ satisfies the Lipschitz-type condition with constants $c_{1}$ and $c_{2}$. Let $f\left(\eta_{n}, u_{n+1}\right)-$ $f\left(\eta_{n}, v_{n}\right)-f\left(v_{n}, u_{n+1}\right)>0$, such that

$$
\begin{aligned}
\frac{\mu\left(\left\|\eta_{n}-v_{n}\right\|^{2}+\left\|u_{n+1}-v_{n}\right\|^{2}\right)}{2\left[f\left(\eta_{n}, u_{n+1}\right)-f\left(\eta_{n}, v_{n}\right)-f\left(v_{n}, u_{n+1}\right)\right]} & \geq \frac{\mu\left(\left\|\eta_{n}-v_{n}\right\|^{2}+\left\|u_{n+1}-v_{n}\right\|^{2}\right)}{2\left[c_{1}\left\|\eta_{n}-v_{n}\right\|^{2}+c_{2}\left\|u_{n+1}-v_{n}\right\|^{2}\right]} \\
& \geq \frac{\mu}{2 \max \left\{c_{1}, c_{2}\right\}} .
\end{aligned}
$$

The above implies that the sequence $\left\{\lambda_{n}\right\}$ has a lower bound $\min \left\{\frac{\mu}{2 \max \left\{c_{1}, c_{2}\right\}}, \lambda_{0}\right\}$. Moreover, there exists a real number $\lambda>0$, such that $\lim _{n \rightarrow \infty} \lambda_{n}=\lambda$. 
Remark 1. Due to the summability of $\sum_{n=0}^{+\infty} \epsilon_{n}$, Expression (5) implies that:

$$
\sum_{n=1}^{\infty} \vartheta_{n}\left\|u_{n}-u_{n-1}\right\| \leq \sum_{n=1}^{\infty} \overline{\vartheta_{n}}\left\|u_{n}-u_{n-1}\right\| \leq \sum_{n=1}^{\infty} \vartheta\left\|u_{n}-u_{n-1}\right\|<\infty
$$

which implies that:

$$
\lim _{n \rightarrow \infty} \vartheta\left\|u_{n}-u_{n-1}\right\|=0
$$

Lemma 6. Assume that a bifunction $f: \mathcal{H} \times \mathcal{H} \rightarrow \mathcal{R}$ satisfies the conditions (f1)-(f4). For each $u^{*} \in E P(f, \mathcal{C}) \neq \varnothing$, we have

$$
\left\|u_{n+1}-u^{*}\right\|^{2} \leq\left\|\eta_{n}-u^{*}\right\|^{2}-\left(1-\frac{\mu \lambda_{n}}{\lambda_{n+1}}\right)\left\|\eta_{n}-v_{n}\right\|^{2}-\left(1-\frac{\mu \lambda_{n}}{\lambda_{n+1}}\right)\left\|u_{n+1}-v_{n}\right\|^{2} .
$$

Proof. From the value of $u_{n+1}$, we have

$$
0 \in \partial_{2}\left\{\lambda_{n} f\left(v_{n}, y\right)+\frac{1}{2}\left\|\eta_{n}-y\right\|^{2}\right\}\left(u_{n+1}\right)+N_{\mathcal{H}_{n}}\left(u_{n+1}\right)
$$

For some $\omega \in \partial f\left(v_{n}, u_{n+1}\right)$ there exists $\bar{\omega} \in N_{\mathcal{H}_{n}}\left(u_{n+1}\right)$ such that

$$
\lambda_{n} \omega+u_{n+1}-\eta_{n}+\bar{\omega}=0
$$

The above equality implies that

$$
\left\langle\eta_{n}-u_{n+1}, y-u_{n+1}\right\rangle=\lambda_{n}\left\langle\omega, y-u_{n+1}\right\rangle+\left\langle\bar{\omega}, y-u_{n+1}\right\rangle, \forall y \in \mathcal{H}_{n}
$$

Since $\bar{\omega} \in N_{\mathcal{H}_{n}}\left(u_{n+1}\right)$, it follows that $\left\langle\bar{\omega}, y-u_{n+1}\right\rangle \leq 0$, for all $y \in \mathcal{H}_{n}$. Thus, we have

$$
\left\langle\eta_{n}-u_{n+1}, y-u_{n+1}\right\rangle \leq \lambda_{n}\left\langle\omega, y-u_{n+1}\right\rangle, \forall y \in \mathcal{H}_{n}
$$

Further, $\omega \in \partial f\left(v_{n}, u_{n+1}\right)$ and due to the definition of subdifferential, we have

$$
f\left(v_{n}, y\right)-f\left(v_{n}, u_{n+1}\right) \geq\left\langle\omega, y-u_{n+1}\right\rangle, \forall y \in \mathcal{H}
$$

Combining Expressions (9) and (10), we obtain

$$
\lambda_{n} f\left(v_{n}, y\right)-\lambda_{n} f\left(v_{n}, u_{n+1}\right) \geq\left\langle\eta_{n}-u_{n+1}, y-u_{n+1}\right\rangle, \forall y \in \mathcal{H}_{n} .
$$

From the definition of $\mathcal{H}_{n}$, we can write

$$
\lambda_{n}\left\langle\omega_{n}, u_{n+1}-v_{n}\right\rangle \geq\left\langle\eta_{n}-v_{n}, u_{n+1}-v_{n}\right\rangle .
$$

Due to $\omega_{n} \in \partial f\left(\eta_{n}, v_{n}\right)$, we have

$$
f\left(\eta_{n}, y\right)-f\left(\eta_{n}, v_{n}\right) \geq\left\langle\omega_{n}, y-v_{n}\right\rangle, \forall y \in \mathcal{H}
$$

By substituting $y=u_{n+1}$ in the above expression, we have

$$
f\left(\eta_{n}, u_{n+1}\right)-f\left(\eta_{n}, v_{n}\right) \geq\left\langle\omega_{n}, u_{n+1}-v_{n}\right\rangle, \forall y \in \mathcal{H} .
$$

Combining Expressions (12) and (13), we obtain

$$
\lambda_{n}\left\{f\left(\eta_{n}, u_{n+1}\right)-f\left(\eta_{n}, v_{n}\right)\right\} \geq\left\langle\eta_{n}-v_{n}, u_{n+1}-v_{n}\right\rangle .
$$


By substituting $y=u^{*}$ in Expression (11), we have

$$
\lambda_{n} f\left(v_{n}, u^{*}\right)-\lambda_{n} f\left(v_{n}, u_{n+1}\right) \geq\left\langle\eta_{n}-u_{n+1}, u^{*}-u_{n+1}\right\rangle .
$$

Since $u^{*} \in E P(f, \mathcal{C})$, we have $f\left(u^{*}, v_{n}\right) \geq 0$. From the pseudomonotonicity of bifunction $f$, we obtain $f\left(v_{n}, u^{*}\right) \leq 0$. Hence, it follows from Expression (15) that

$$
\left\langle\eta_{n}-u_{n+1}, u_{n+1}-u^{*}\right\rangle \geq \lambda_{n} f\left(v_{n}, u_{n+1}\right) .
$$

From the definition of $\lambda_{n+1}$, we obtain

$$
f\left(\eta_{n}, u_{n+1}\right)-f\left(\eta_{n}, v_{n}\right)-f\left(v_{n}, u_{n+1}\right) \leq \frac{\mu\left\|\eta_{n}-v_{n}\right\|^{2}+\mu\left\|u_{n+1}-v_{n}\right\|^{2}}{2 \lambda_{n+1}}
$$

From Expressions (16) and (17), we have

$$
\begin{aligned}
\left\langle\eta_{n}-u_{n+1}, u_{n+1}-u^{*}\right\rangle \geq & \lambda_{n}\left\{f\left(\eta_{n}, u_{n+1}\right)-f\left(\eta_{n}, v_{n}\right)\right\} \\
& -\frac{\mu \lambda_{n}}{2 \lambda_{n+1}}\left\|\eta_{n}-v_{n}\right\|^{2}-\frac{\mu \lambda_{n}}{2 \lambda_{n+1}}\left\|u_{n+1}-v_{n}\right\|^{2} .
\end{aligned}
$$

Combining Expressions (14) and (18), we obtain

$$
\begin{aligned}
\left\langle\eta_{n}-u_{n+1}, u_{n+1}-u^{*}\right\rangle \geq & \left\langle\eta_{n}-v_{n}, u_{n+1}-v_{n}\right\rangle \\
& -\frac{\mu \lambda_{n}}{2 \lambda_{n+1}}\left\|\eta_{n}-v_{n}\right\|^{2}-\frac{\mu \lambda_{n}}{2 \lambda_{n+1}}\left\|u_{n+1}-v_{n}\right\|^{2} .
\end{aligned}
$$

We have the following formulas:

$$
\begin{gathered}
-2\left\langle\eta_{n}-u_{n+1}, u_{n+1}-u^{*}\right\rangle=-\left\|\eta_{n}-u^{*}\right\|^{2}+\left\|u_{n+1}-\eta_{n}\right\|^{2}+\left\|u_{n+1}-u^{*}\right\|^{2} . \\
2\left\langle v_{n}-\eta_{n}, v_{n}-u_{n+1}\right\rangle=\left\|\eta_{n}-v_{n}\right\|^{2}+\left\|u_{n+1}-v_{n}\right\|^{2}-\left\|\eta_{n}-u_{n+1}\right\|^{2} .
\end{gathered}
$$

Combining the relations (19)-(21), we get

$$
\left\|u_{n+1}-u^{*}\right\|^{2} \leq\left\|\eta_{n}-u^{*}\right\|^{2}-\left(1-\frac{\mu \lambda_{n}}{\lambda_{n+1}}\right)\left\|\eta_{n}-v_{n}\right\|^{2}-\left(1-\frac{\mu \lambda_{n}}{\lambda_{n+1}}\right)\left\|u_{n+1}-v_{n}\right\|^{2} .
$$

Theorem 1. Assume that a bifunction $f: \mathcal{H} \times \mathcal{H} \rightarrow \mathcal{R}$ satisfies the conditions (f1)-(f4) and $u^{*}$ belongs to solution set $E P(f, \mathcal{C})$. Then, the sequences $\left\{\eta_{n}\right\},\left\{u_{n}\right\}$ and $\left\{v_{n}\right\}$ generated by Algorithm 1 converge weakly to the $u^{*}$ solution of the problem (1). In addition, $\lim _{n \rightarrow \infty} P_{E P(f, \mathcal{C})}\left(u_{n}\right)=u^{*}$.

Proof. Since $\lambda_{n} \rightarrow \lambda$, there exists a fixed number $\epsilon \in(0,1-\mu)$ such that

$$
\lim _{n \rightarrow \infty}\left(1-\frac{\mu \lambda_{n}}{\lambda_{n+1}}\right)=1-\mu>\epsilon>0 .
$$

Thus, there is a finite number $n_{1} \in \mathcal{N}$ such that

$$
\left(1-\frac{\mu \lambda_{n}}{\lambda_{n+1}}\right)>\epsilon>0, \forall n \geq n_{1} .
$$


By Lemma 6, we obtain

$$
\left\|u_{n+1}-u^{*}\right\|^{2} \leq\left\|\eta_{n}-u^{*}\right\|^{2}, \forall n \geq n_{1} .
$$

From the definition of $\eta_{n}$ in Algorithm 1, we have

$$
\begin{aligned}
\left\|\eta_{n}-u^{*}\right\|^{2} & =\left\|u_{n}+\vartheta_{n}\left(u_{n}-u_{n-1}\right)-u^{*}\right\|^{2} \\
& =\left\|\left(1+\vartheta_{n}\right)\left(u_{n}-u^{*}\right)-\vartheta_{n}\left(u_{n-1}-u^{*}\right)\right\|^{2} \\
& =\left(1+\vartheta_{n}\right)\left\|u_{n}-u^{*}\right\|^{2}-\vartheta_{n}\left\|u_{n-1}-u^{*}\right\|^{2}+\vartheta_{n}\left(1+\vartheta_{n}\right)\left\|u_{n}-u_{n-1}\right\|^{2} \\
& \leq\left(1+\vartheta_{n}\right)\left\|u_{n}-u^{*}\right\|^{2}-\vartheta_{n}\left\|u_{n-1}-u^{*}\right\|^{2}+2 \vartheta\left\|u_{n}-u_{n-1}\right\|^{2} .
\end{aligned}
$$

Expression (23) can be written as

$$
\left\|u_{n+1}-u^{*}\right\|^{2} \leq\left(1+\vartheta_{n}\right)\left\|u_{n}-u^{*}\right\|^{2}-\vartheta_{n}\left\|u_{n-1}-u^{*}\right\|^{2}+2 \vartheta\left\|u_{n}-u_{n-1}\right\|^{2}, \quad \forall n \geq n_{1} .
$$

From the definition of the $\eta_{n}$, we also have

$$
\left\|\eta_{n}-u^{*}\right\|=\left\|u_{n}+\vartheta_{n}\left(u_{n}-u_{n-1}\right)-u^{*}\right\| \leq\left\|u_{n}-u^{*}\right\|+\vartheta_{n}\left\|u_{n}-u_{n-1}\right\| .
$$

Combining relations (23) and (27), we obtain

$$
\left\|u_{n+1}-u^{*}\right\| \leq\left\|u_{n}-u^{*}\right\|+\vartheta\left\|u_{n}-u_{n-1}\right\|, \quad \forall n \geq n_{1} .
$$

By using Lemma 4 with (7) and (28), we have

$$
\lim _{n \rightarrow \infty}\left\|u_{n}-u^{*}\right\|=l, \text { for some finite } l \geq 0 .
$$

From Equality (8), we have

$$
\lim _{n \rightarrow \infty}\left\|u_{n}-u_{n-1}\right\|=0 .
$$

By letting $n \rightarrow \infty$ in Expression (24), we obtain

$$
\lim _{n \rightarrow \infty}\left\|\eta_{n}-u^{*}\right\|=l .
$$

From Lemma 6 and Expression (25), we have

$$
\begin{aligned}
& \left\|u_{n+1}-u^{*}\right\|^{2} \\
& \leq\left(1+\vartheta_{n}\right)\left\|u_{n}-u^{*}\right\|^{2}-\vartheta_{n}\left\|u_{n-1}-u^{*}\right\|^{2}+2 \vartheta\left\|u_{n}-u_{n-1}\right\|^{2} \\
& \quad-\left(1-\frac{\mu \lambda_{n}}{\lambda_{n+1}}\right)\left\|\eta_{n}-v_{n}\right\|^{2}-\left(1-\frac{\mu \lambda_{n}}{\lambda_{n+1}}\right)\left\|u_{n+1}-v_{n}\right\|^{2},
\end{aligned}
$$

which further implies that (for $n \geq n_{1}$ )

$$
\begin{aligned}
& \epsilon\left\|\eta_{n}-v_{n}\right\|^{2}+\epsilon\left\|v_{n}-u_{n+1}\right\|^{2} \\
& \leq\left\|u_{n}-u^{*}\right\|^{2}-\left\|u_{n+1}-u^{*}\right\|^{2}+\vartheta_{n}\left(\left\|u_{n}-u^{*}\right\|^{2}-\left\|u_{n-1}-u^{*}\right\|^{2}\right)+2 \vartheta\left\|u_{n}-u_{n-1}\right\|^{2} .
\end{aligned}
$$

By letting $n \rightarrow \infty$ in (33), we obtain

$$
\lim _{n \rightarrow \infty}\left\|\eta_{n}-v_{n}\right\|=\lim _{n \rightarrow \infty}\left\|v_{n}-u_{n+1}\right\|=0 .
$$


By using the Cauchy inequality and Expression (34), we obtain

$$
\lim _{n \rightarrow \infty}\left\|\eta_{n}-u_{n+1}\right\| \leq \lim _{n \rightarrow \infty}\left\|\eta_{n}-v_{n}\right\|+\lim _{n \rightarrow \infty}\left\|u_{n+1}-v_{n}\right\|=0 .
$$

From Expressions (31) and (34), we also obtain

$$
\lim _{n \rightarrow \infty}\left\|v_{n}-u^{*}\right\|=l .
$$

It follows from Expressions (29), (31), and (36) that the sequences $\left\{\eta_{n}\right\},\left\{u_{n}\right\}$, and $\left\{v_{n}\right\}$ are bounded. Next, we need to use Lemma 3 , for it is compulsory to prove that all sequential weak cluster limit points of the sequence $\left\{u_{n}\right\}$ belong to the solution set $E P(f, \mathcal{C})$. Assume that $z$ is any weak cluster limit point of the sequence $\left\{u_{n}\right\}$, i.e., there exists a subsequence $\left\{u_{n_{k}}\right\}$ of $\left\{u_{n}\right\}$ such that $u_{n_{k}} \rightarrow z$. Since $\left\|u_{n}-v_{n}\right\| \rightarrow 0$, it follows that $\left\{v_{n_{k}}\right\}$ also weakly converges to $z$ and so $z \in \mathcal{C}$. Now, it remains to prove that $z \in E P(f, \mathcal{C})$. By Expression (11), the definition of $\lambda_{n+1}$, and (14), we have

$$
\begin{aligned}
\lambda_{n_{k}} f\left(v_{n_{k}}, y\right) \geq & \lambda_{n_{k}} f\left(v_{n_{k}}, u_{n_{k}+1}\right)+\left\langle\eta_{n_{k}}-u_{n_{k}+1}, y-u_{n_{k}+1}\right\rangle \\
\geq & \lambda_{n_{k}} f\left(\eta_{n_{k}}, u_{n_{k+1}}\right)-\lambda_{n_{k}} f\left(\eta_{n_{k}}, v_{n_{k}}\right)-\frac{\mu \lambda_{n_{k}}}{2 \lambda_{n_{k}+1}}\left\|\eta_{n_{k}}-v_{n_{k}}\right\|^{2} \\
& -\frac{\mu \lambda_{n_{k}}}{2 \lambda_{n_{k}+1}}\left\|v_{n_{k}}-u_{n_{k}+1}\right\|^{2}+\left\langle\eta_{n_{k}}-u_{n_{k}+1}, y-u_{n_{k}+1}\right\rangle \\
\geq & \left\langle\eta_{n_{k}}-v_{n_{k}}, u_{n_{k}+1}-v_{n_{k}}\right\rangle-\frac{\mu \lambda_{n_{k}}}{2 \lambda_{n_{k}+1}}\left\|\eta_{n_{k}}-v_{n_{k}}\right\|^{2} \\
& -\frac{\mu \lambda_{n_{k}}}{2 \lambda_{n_{k}+1}}\left\|v_{n_{k}}-u_{n_{k}+1}\right\|^{2}+\left\langle\eta_{n_{k}}-u_{n_{k}+1}, y-u_{n_{k}+1}\right\rangle,
\end{aligned}
$$

where $y \in \mathcal{H}_{n}$. It follows from (30), (34), (35), and the boundedness of $\left\{u_{n}\right\}$ that the right hand side tends to zero. Due to $\lambda_{n_{k}}>0$, condition (f3), and $v_{n_{k}} \rightarrow z$, we have

$$
0 \leq \limsup _{k \rightarrow \infty} f\left(v_{n_{k}}, y\right) \leq f(z, y), \forall y \in \mathcal{H}_{n} .
$$

Since $\mathcal{C} \subset \mathcal{H}_{n}$, it follows that $f(z, y) \geq 0, \forall y \in \mathcal{C}$. This implies that $z \in E P(f, \mathcal{C})$. Finally, from Lemma 3 , the sequences $\left\{\eta_{n}\right\},\left\{u_{n}\right\}$, and $\left\{v_{n}\right\}$ converge weakly to $u^{*}$ as $n \rightarrow \infty$.

Moreover, the renaming part consists of proving that $\lim _{n \rightarrow \infty} P_{E P(f, \mathcal{C})}\left(u_{n}\right)=u^{*}$. Let $q_{n}:=P_{E P(f, \mathcal{C})}\left(u_{n}\right), \forall n \in \mathcal{N}$. For any $u^{*} \in E P(f, \mathcal{C})$, we have

$$
\left\|q_{n}\right\| \leq\left\|q_{n}-u_{n}\right\|+\left\|u_{n}\right\| \leq\left\|u^{*}-u_{n}\right\|+\left\|u_{n}\right\| .
$$

The above expression implies that the sequence $\left\{q_{n}\right\}$ is bounded. Next, we prove that $\left\{q_{n}\right\}$ is a Cauchy sequence. By Lemma 1(iii) and (27), we have

$$
\left\|u_{n+1}-q_{n+1}\right\| \leq\left\|u_{n+1}-q_{n}\right\| \leq\left\|u_{n}-q_{n}\right\|+\vartheta\left\|u_{n}-u_{n-1}\right\|, \forall n \geq n_{1} .
$$

Lemma 4 provides the existence of $\lim _{n \rightarrow \infty}\left\|u_{n}-q_{n}\right\|$. From Expression (27) for all $m>n \geq n_{1}$, we have

$$
\begin{aligned}
\left\|q_{n}-u_{m}\right\| & \leq\left\|q_{n}-u_{m-1}\right\|+\vartheta\left\|u_{n}-u_{n-1}\right\| \\
& \leq \cdots \leq\left\|q_{n}-u_{n}\right\|+\vartheta \sum_{k=n}^{m-1}\left\|u_{n}-u_{n-1}\right\| .
\end{aligned}
$$


Suppose that $q_{m}, q_{n} \in E P(f, \mathcal{C})$ for $m>n \geq n_{1}$. By using Lemma 1(i) and Expression (40), we have

$$
\begin{aligned}
& \left\|q_{n}-q_{m}\right\|^{2} \\
& \leq\left\|q_{n}-u_{m}\right\|^{2}-\left\|q_{m}-u_{m}\right\|^{2} \\
& \leq\left\|q_{n}-u_{n}\right\|^{2}+\left(\vartheta \sum_{k=n}^{m-1}\left\|u_{n}-u_{n-1}\right\|\right)^{2}+2 \vartheta\left\|q_{n}-u_{n}\right\| \sum_{k=n}^{m-1}\left\|u_{n}-u_{n-1}\right\|-\left\|q_{m}-u_{m}\right\|^{2} .
\end{aligned}
$$

The existence of $\lim _{n \rightarrow \infty}\left\|u_{n}-q_{n}\right\|$ and the summability of the series $\sum_{n}\left\|u_{n}-u_{n-1}\right\|$ imply that $\lim _{n \rightarrow \infty}\left\|q_{n}-q_{m}\right\|=0$, for all $m>n$. As a result, $\left\{q_{n}\right\}$ is a Cauchy sequence and due to the closeness of a solution set $E P(f, \mathcal{C})$ the sequence $\left\{q_{n}\right\}$ strongly converges to $q^{*} \in E P(f, \mathcal{C})$. Next, we show that $q^{*}=u^{*}$. Due to Lemma 1(ii) and $u^{*}, q^{*} \in E P(f, \mathcal{C})$, we can write

$$
\left\langle u_{n}-q_{n}, u^{*}-q_{n}\right\rangle \leq 0 .
$$

Due to $q_{n} \rightarrow q^{*}$ and $u_{n} \rightarrow u^{*}$, we obtain

$$
\left\langle u^{*}-q^{*}, u^{*}-q^{*}\right\rangle \leq 0
$$

which gives that $u^{*}=q^{*}=\lim _{n \rightarrow \infty} P_{E P(f, \mathcal{C})}\left(u_{n}\right)$.

\section{Applications to Solve Fixed Point Problems}

Now, consider the applications of our results from Section 3 to solve fixed-point problems involving $\kappa$-strict pseudo-contraction. A mapping $T: \mathcal{C} \rightarrow \mathcal{C}$ is said to be

(i) $\mathcal{K}$-strict pseudo-contraction [45] on $\mathcal{C}$ if

$$
\left\|T z_{1}-T z_{2}\right\|^{2} \leq\left\|z_{1}-z_{2}\right\|^{2}+\kappa\left\|\left(z_{1}-T z_{1}\right)-\left(z_{2}-T z_{2}\right)\right\|^{2}, \forall z_{1}, z_{2} \in \mathcal{C},
$$

which is equivalent to

$$
\left\langle T z_{1}-T z_{2}, z_{1}-z_{2}\right\rangle \leq\left\|z_{1}-z_{2}\right\|^{2}-\frac{1-\kappa}{2}\left\|\left(z_{1}-T z_{1}\right)-\left(z_{2}-T z_{2}\right)\right\|^{2}, \forall z_{1}, z_{2} \in \mathcal{C} ;
$$

(ii) sequentially weakly continuous on $\mathcal{C}$ if

$$
T\left(u_{n}\right) \rightarrow T(p) \text { for every sequence in } \mathcal{C} \text { satisfying } u_{n} \rightarrow p \text { (weakly converges). }
$$

The fixed point problem for a mapping $T: \mathcal{C} \rightarrow \mathcal{C}$ is formulated in the following way:

Find $u^{*} \in \mathcal{C}$ such that $T\left(u^{*}\right)=u^{*}$.

Note: If we define bifunction $f(x, y)=\langle x-T x, y-x\rangle, \forall x, y \in \mathcal{C}$. Then, the equilibrium problem (1) converts into the fixed point problem with $2 c_{1}=2 c_{2}=\frac{3-2 \kappa}{1-\kappa}$. From the value of $v_{n}$ in Algorithm 1, we have 


$$
\begin{aligned}
v_{n} & =\underset{y \in \mathcal{C}}{\arg \min }\left\{\lambda_{n} f\left(\eta_{n}, y\right)+\frac{1}{2}\left\|\eta_{n}-y\right\|^{2}\right\} \\
& =\underset{y \in \mathcal{C}}{\arg \min }\left\{\lambda_{n}\left\langle\eta_{n}-T\left(\eta_{n}\right), y-\eta_{n}\right\rangle+\frac{1}{2}\left\|\eta_{n}-y\right\|^{2}\right\} \\
& =\underset{y \in \mathcal{C}}{\arg \min }\left\{\lambda_{n}\left\langle\eta_{n}-T\left(\eta_{n}\right), y-\eta_{n}\right\rangle+\frac{1}{2}\left\|\eta_{n}-y\right\|^{2}+\frac{\lambda_{n}^{2}}{2}\left\|\eta_{n}-T\left(\eta_{n}\right)\right\|^{2}-\frac{\lambda_{n}^{2}}{2}\left\|\eta_{n}-T\left(\eta_{n}\right)\right\|^{2}\right\} \\
& =\underset{y \in \mathcal{C}}{\arg \min }\left\{\frac{1}{2}\left\|y-\eta_{n}+\lambda_{n}\left(\eta_{n}-T\left(\eta_{n}\right)\right)\right\|^{2}\right\} \\
& =P_{\mathcal{C}}\left[\eta_{n}-\lambda_{n}\left(\eta_{n}-T\left(\eta_{n}\right)\right)\right]=P_{\mathcal{C}}\left[\left(1-\lambda_{n}\right) \eta_{n}+\lambda_{n} T\left(\eta_{n}\right)\right] .
\end{aligned}
$$

Since $\omega_{n} \in \partial_{2} f\left(\eta_{n}, v_{n}\right)$, it follows from the definition of the subdifferential that we have

$$
\begin{aligned}
\left\langle\omega_{n}, y-v_{n}\right\rangle & \leq\left\langle\eta_{n}-T\left(\eta_{n}\right), y-\eta_{n}\right\rangle-\left\langle\eta_{n}-T\left(\eta_{n}\right), v_{n}-\eta_{n}\right\rangle, \forall y \in \mathcal{H} \\
& \leq\left\langle\eta_{n}-T\left(\eta_{n}\right), y-v_{n}\right\rangle, \forall y \in \mathcal{H},
\end{aligned}
$$

and consequently $0 \leq\left\langle\eta_{n}-T\left(\eta_{n}\right)-\omega_{n}, y-v_{n}\right\rangle$. This implies that

$$
\begin{aligned}
& \left\langle\left(1-\lambda_{n}\right) \eta_{n}+\lambda_{n} T\left(\eta_{n}\right)-v_{n}, y-v_{n}\right\rangle \\
& \leq\left\langle\left(1-\lambda_{n}\right) \eta_{n}+\lambda_{n} T\left(\eta_{n}\right)-v_{n}, y-v_{n}\right\rangle+\lambda_{n}\left\langle\eta_{n}-T\left(\eta_{n}\right)-\omega_{n}, y-v_{n}\right\rangle \\
& \leq\left\langle\eta_{n}-\lambda_{n} \omega_{n}-v_{n}, y-v_{n}\right\rangle .
\end{aligned}
$$

Similarly to Expression (45), we obtain

$$
u_{n+1}=P_{\mathcal{H}_{n}}\left[\eta_{n}-\lambda_{n}\left(v_{n}-T\left(v_{n}\right)\right)\right] .
$$

As a consequence of the results in Section 3, we have the following fixed point theorem:

Corollary 1. Let $\mathcal{C}$ be a subset of a Hilbert space $\mathcal{H}$ and $T: \mathcal{C} \rightarrow \mathcal{C}$ be a $\kappa$-strict pseudocontraction and weakly continuous with Fix $(T) \neq \varnothing$. The sequences $\eta_{n}, u_{n}$, and $v_{n}$ are generated in the following way:

(i) Fix $u_{-1}, u_{0} \in \mathcal{C}, \lambda_{0}>0, \mu \in(0,1)$ and $\vartheta \in[0,1)$ with a sequence $\left\{\epsilon_{n}\right\} \subset[0,+\infty)$ such that

$$
\sum_{n=0}^{+\infty} \epsilon_{n}<+\infty
$$

(ii) Choose $\vartheta_{n}$ such that $0 \leq \vartheta_{n} \leq \bar{\vartheta}_{n}$ and

$$
\overline{\vartheta_{n}}= \begin{cases}\min \left\{\vartheta, \frac{\epsilon_{n}}{\left\|u_{n}-u_{n-1}\right\|}\right\} & \text { if } u_{n} \neq u_{n-1} \\ \vartheta & \text { otherwise. }\end{cases}
$$

(iii) Evaluate

$$
\left\{\begin{array}{l}
\eta_{n}=u_{n}+\vartheta_{n}\left(u_{n}-u_{n-1}\right) \\
v_{n}=P_{\mathcal{C}}\left[\eta_{n}-\lambda_{n}\left(\eta_{n}-T\left(\eta_{n}\right)\right)\right] \\
u_{n+1}=P_{\mathcal{H}_{n}}\left[\eta_{n}-\lambda_{n}\left(v_{n}-T\left(v_{n}\right)\right)\right]
\end{array}\right.
$$

where $\mathcal{H}_{n}=\left\{z \in \mathcal{H}:\left\langle\left(1-\lambda_{n}\right) \eta_{n}+\lambda_{n} T\left(\eta_{n}\right)-v_{n}, z-v_{n}\right\rangle \leq 0\right\}$.

(iv) Set $d_{2}=\left\langle\left(\eta_{n}-v_{n}\right)-\left(T\left(\eta_{n}\right)-T\left(v_{n}\right)\right), u_{n+1}-v_{n}\right\rangle$ and revise the stepsize $\lambda_{n+1}$ in the following way:

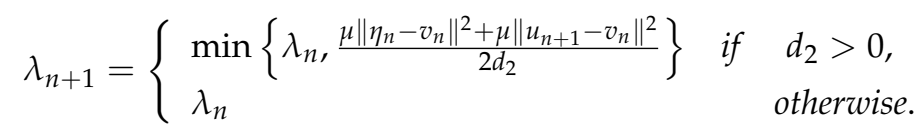


Then, sequences $\left\{\eta_{n}\right\},\left\{u_{n}\right\}$, and $\left\{v_{n}\right\}$ weakly converge to $u^{*} \in \operatorname{Fix}(T)$.

\section{Application to Solve Variational Inequality Problems}

Now, consider the applications of our results from in Section 3 to solve variational inequality problems involving a pseudomonotone and Lipschitz-type continuous operator. An operator $K: \mathcal{H} \rightarrow \mathcal{H}$ is said to be

(i) L-Lipschitz continuous on $\mathcal{C}$ if

$$
\left\|K\left(z_{1}\right)-K\left(z_{2}\right)\right\| \leq L\left\|z_{1}-z_{2}\right\|, \forall z_{1}, z_{2} \in \mathcal{C} ;
$$

(ii) pseudomonotone on $\mathcal{C}$ if

$$
\left\langle K\left(z_{1}\right), z_{2}-z_{1}\right\rangle \geq 0 \Longrightarrow\left\langle K\left(z_{2}\right), z_{1}-z_{2}\right\rangle \leq 0, \forall z_{1}, z_{2} \in \mathcal{C} .
$$

The variational inequality problem for a operator $K: \mathcal{H} \rightarrow \mathcal{H}$ is formulated in the following way:

$$
\text { Find } u^{*} \in \mathcal{C} \text { such that }\left\langle K\left(u^{*}\right), y-u^{*}\right\rangle \geq 0, \forall y \in \mathcal{C} \text {. }
$$

Note: If we define a bifunction $f(x, y):=\langle K(x), y-x\rangle, \forall x, y \in \mathcal{C}$. Thus, the equilibrium problem (1) translates into a variational inequality problem with $L=2 c_{1}=2 c_{2}$. From the value of $v_{n}$, we have

$$
\begin{aligned}
v_{n} & =\underset{y \in \mathcal{C}}{\arg \min }\left\{\lambda_{n} f\left(\eta_{n}, y\right)+\frac{1}{2}\left\|\eta_{n}-y\right\|^{2}\right\} \\
& =\underset{y \in \mathcal{C}}{\arg \min }\left\{\lambda_{n}\left\langle K\left(\eta_{n}\right), y-\eta_{n}\right\rangle+\frac{1}{2}\left\|\eta_{n}-y\right\|^{2}+\frac{\lambda_{n}^{2}}{2}\left\|K\left(\eta_{n}\right)\right\|^{2}-\frac{\lambda_{n}^{2}}{2}\left\|K\left(\eta_{n}\right)\right\|^{2}\right\} \\
& =\underset{y \in \mathcal{C}}{\arg \min }\left\{\frac{1}{2}\left\|y-\left(\eta_{n}-\lambda_{n} K\left(\eta_{n}\right)\right)\right\|^{2}\right\} \\
& =P_{\mathcal{C}}\left[\eta_{n}-\lambda_{n} K\left(\eta_{n}\right)\right] .
\end{aligned}
$$

Since $\omega_{n} \in \partial_{2} f\left(\eta_{n}, v_{n}\right)$, it follows from the subdifferential definition that we have

$$
\begin{aligned}
\left\langle\omega_{n}, y-v_{n}\right\rangle & \leq\left\langle K\left(\eta_{n}\right), y-\eta_{n}\right\rangle-\left\langle K\left(\eta_{n}\right), v_{n}-\eta_{n}\right\rangle, \forall y \in \mathcal{H} \\
& =\left\langle K\left(\eta_{n}\right), y-v_{n}\right\rangle, \forall y \in \mathcal{H}
\end{aligned}
$$

and consequently $0 \leq\left\langle K\left(\eta_{n}\right)-\omega_{n}, y-v_{n}\right\rangle$. This implies that

$$
\begin{aligned}
& \left\langle\eta_{n}-\lambda_{n} K\left(\eta_{n}\right)-v_{n}, y-v_{n}\right\rangle \\
& \leq\left\langle\eta_{n}-\lambda_{n} K\left(\eta_{n}\right)-v_{n}, y-v_{n}\right\rangle+\lambda_{n}\left\langle K\left(\eta_{n}\right)-\omega_{n}, y-v_{n}\right\rangle \\
& \leq\left\langle\eta_{n}-\lambda_{n} \omega_{n}-v_{n}, y-v_{n}\right\rangle .
\end{aligned}
$$

In similar way to Expression (52), we have

$$
u_{n+1}=P_{\mathcal{H}_{n}}\left[\eta_{n}-\lambda_{n} K\left(v_{n}\right)\right] .
$$

Suppose that $K$ satisfies the following conditions:

(K1) $\quad K$ is pseudomonotone on $\mathcal{C}$ with $V I(K, \mathcal{C}) \neq \varnothing$;

(K2) $K$ is $L$-Lipschitz continuous on $\mathcal{C}$ with $L>0$;

(K3) $\quad \limsup \left\langle K\left(u_{n}\right), y-u_{n}\right\rangle \leq\langle K(p), y-p\rangle, \forall y \in \mathcal{C}$ and $\left\{u_{n}\right\} \subset \mathcal{C}$ satisfying $u_{n} \rightarrow p$. 
Corollary 2. Assume that a operator $K: \mathcal{C} \rightarrow \mathcal{H}$ satisfies the conditions (K1)-(K3) and that the sequences $\left\{\eta_{n}\right\},\left\{u_{n}\right\}$, and $\left\{v_{n}\right\}$ are generated in the following way:

(i) Choose $u_{-1}, u_{0} \in \mathcal{C}, \lambda_{0}>0, \mu \in(0,1)$ and $\vartheta \in[0,1)$ with $\left\{\epsilon_{n}\right\} \subset[0,+\infty)$ such that

$$
\sum_{n=0}^{+\infty} \epsilon_{n}<+\infty
$$

(ii) Choose $\vartheta_{n}$ satisfying $0 \leq \vartheta_{n} \leq \bar{\vartheta}_{n}$ such that

$$
\bar{\vartheta}_{n}= \begin{cases}\min \left\{\vartheta, \frac{\epsilon_{n}}{\left\|u_{n}-u_{n-1}\right\|}\right\} & \text { if } u_{n} \neq u_{n-1} \\ \vartheta & \text { otherwise. }\end{cases}
$$

(iii) Set $\eta_{n}=u_{n}+\vartheta_{n}\left(u_{n}-u_{n-1}\right)$ and compute

$$
\left\{\begin{array}{l}
v_{n}=P_{\mathcal{C}}\left[\eta_{n}-\lambda_{n} K\left(\eta_{n}\right)\right], \\
u_{n+1}=P_{\mathcal{H}_{n}}\left[\eta_{n}-\lambda_{n} K\left(v_{n}\right)\right]
\end{array}\right.
$$

where $\mathcal{H}_{n}=\left\{z \in \mathcal{H}:\left\langle\eta_{n}-\lambda_{n} K\left(\eta_{n}\right)-v_{n}, z-v_{n}\right\rangle \leq 0\right\}$.

(iv) Set $d_{3}=\left\langle K\left(\eta_{n}\right)-K\left(v_{n}\right), u_{n+1}-v_{n}\right\rangle$ and stepsize $\lambda_{n+1}$ is revised in the following way:

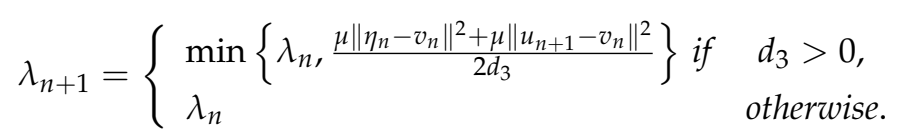

Then, the sequences $\left\{\eta_{n}\right\},\left\{u_{n}\right\}$, and $\left\{v_{n}\right\}$ weakly converge to $u^{*} \in V I(K, \mathcal{C})$.

\section{Numerical Experiments}

The computational results are presented in this section to illustrate the effectiveness of our proposed Algorithm 1 (EiEGM) compared to Algorithm 1 (iEGM) in [39]. The MATLAB program was operated on a PC (with Intel(R) Core(TM)i3-4010U CPU @ 1.70GHz 1.70GHz, RAM 4.00 GB) in MATLAB version 9.5 (R2018b). We used the built-in MATLAB fmincon function to solve the minimization problems.

Example 1. Let $f: \mathcal{C} \times \mathcal{C} \rightarrow \mathcal{R}$ be defined by

$$
f(u, v)=\sum_{i=2}^{5}\left(v_{i}-u_{i}\right)\|u\|, \forall u, v \in \mathcal{R}^{5},
$$

where $\mathcal{C}=\left\{\left(u_{1}, \cdots, u_{5}\right): u_{1} \geq-1, u_{i} \geq 1, i=2, \cdots, 5\right\}$. The bifunction $f$ is Lipschitz-type continuous operator with constants $c_{1}=c_{2}=2$, and it satisfies conditions (f1)-(f4). To evaluate the best possible value of the control parameters, two tests were performed taking into consideration the variation of the control parameters $\lambda, \lambda_{0}$ and inertial factor $\vartheta$. The numerical results are shown in the Tables 1 and 2 by choosing $u_{-1}=u_{0}=(2,3,2,5,5), \mu=0.33$ and $D_{n}=\left\|\eta_{n}-v_{n}\right\| \leq \epsilon=10^{-4}$. 
Table 1. Example 1: Algorithm 1 numerical comparison with Algorithm 1 in [39].

\begin{tabular}{ccccccc}
\hline & \multicolumn{4}{c}{ Number of Iterations } & \multicolumn{2}{c}{ Execution Time in Seconds } \\
\hline $\boldsymbol{\vartheta}$ & $\lambda$ & $\boldsymbol{\lambda}_{\mathbf{0}}$ & iEGM & EiEGM & iEGM & EiEGM \\
\hline 0.45 & 0.22 & 1.00 & 12 & 7 & 0.8675 & 0.5324 \\
0.45 & 0.16 & 0.80 & 13 & 7 & 0.8815 & 0.5423 \\
0.45 & 0.10 & 0.60 & 17 & 7 & 1.0915 & 0.5212 \\
0.45 & 0.05 & 0.40 & 21 & 8 & 1.4119 & 0.5567 \\
0.45 & 0.01 & 0.20 & 25 & 9 & 1.7229 & 0.5881 \\
\hline
\end{tabular}

Table 2. Example 1: Algorithm 1 numerical comparison with Algorithm 1 in [39].

\begin{tabular}{ccccccc}
\hline & \multicolumn{3}{c}{ Number of Iterations } & \multicolumn{2}{c}{ Execution Time in Seconds } \\
\hline $\boldsymbol{\vartheta}$ & $\lambda$ & $\lambda_{\mathbf{0}}$ & iEGM & EiEGM & iEGM & EiEGM \\
\hline 0.95 & 0.20 & 0.50 & 19 & 7 & 1.1482 & 0.4911 \\
0.75 & 0.20 & 0.50 & 14 & 7 & 0.9676 & 0.5026 \\
0.55 & 0.20 & 0.50 & 13 & 7 & 0.9654 & 0.4991 \\
0.35 & 0.20 & 0.50 & 12 & 8 & 0.9123 & 0.5092 \\
0.15 & 0.20 & 0.50 & 17 & 9 & 1.0715 & 0.5098 \\
\hline
\end{tabular}

Example 2. Consider the Nash-Cournot equilibrium model that appeared in the paper [7]. The bifunction $f$ has been defined in the following way:

$$
f(u, v)=\langle A u+B v+c, v-u\rangle
$$

where $c \in \mathcal{R}^{5}$ and matrices $A, B$ are

$$
A=\left(\begin{array}{ccccc}
3.1 & 2 & 0 & 0 & 0 \\
2 & 3.6 & 0 & 0 & 0 \\
0 & 0 & 3.5 & 2 & 0 \\
0 & 0 & 2 & 3.3 & 0 \\
0 & 0 & 0 & 0 & 3
\end{array}\right) \quad B=\left(\begin{array}{ccccc}
1.6 & 1 & 0 & 0 & 0 \\
1 & 1.6 & 0 & 0 & 0 \\
0 & 0 & 1.5 & 1 & 0 \\
0 & 0 & 1 & 1.5 & 0 \\
0 & 0 & 0 & 0 & 2
\end{array}\right) \quad c=\left(\begin{array}{c}
1 \\
-2 \\
-1 \\
2 \\
-1
\end{array}\right)
$$

while Lipschitz constants $c_{1}=c_{2}=\frac{1}{2}\|A-B\|$ (see for more details $[7,46,47]$ ). The set $\mathcal{C} \subset \mathcal{R}^{5}$ is $\mathcal{C}:=\left\{u \in \mathcal{R}^{5}:-5 \leq u_{i} \leq 5\right\}$. Figures 1 and 2 and Table 3 report the numerical results by choosing $u_{-1}=u_{0}=(1, \cdots, 1), \mu=0.33$ and $\epsilon=10^{-6}$.

Table 3. Figures 1 and 2: Algorithm 1 numerical comparison with Algorithm 1 in [39].

\begin{tabular}{ccccclc}
\hline & \multicolumn{4}{c}{ Number of Iterations } & \multicolumn{2}{l}{ Execution Time in Seconds } \\
\hline $\boldsymbol{\vartheta}$ & $\lambda$ & $\lambda_{\mathbf{0}}$ & iEGM & EiEGM & iEGM & EiEGM \\
\hline 0.50 & 0.05 & 0.15 & 98 & 64 & 2.2174 & 1.6342 \\
0.50 & 0.10 & 0.35 & 64 & 50 & 1.6815 & 1.3452 \\
0.50 & 0.15 & 0.55 & 54 & 46 & 1.5712 & 1.2011 \\
0.50 & 0.20 & 0.75 & 50 & 42 & 1.5196 & 1.0845 \\
0.50 & 0.25 & 0.95 & 45 & 38 & 1.3859 & 1.0023 \\
\hline
\end{tabular}




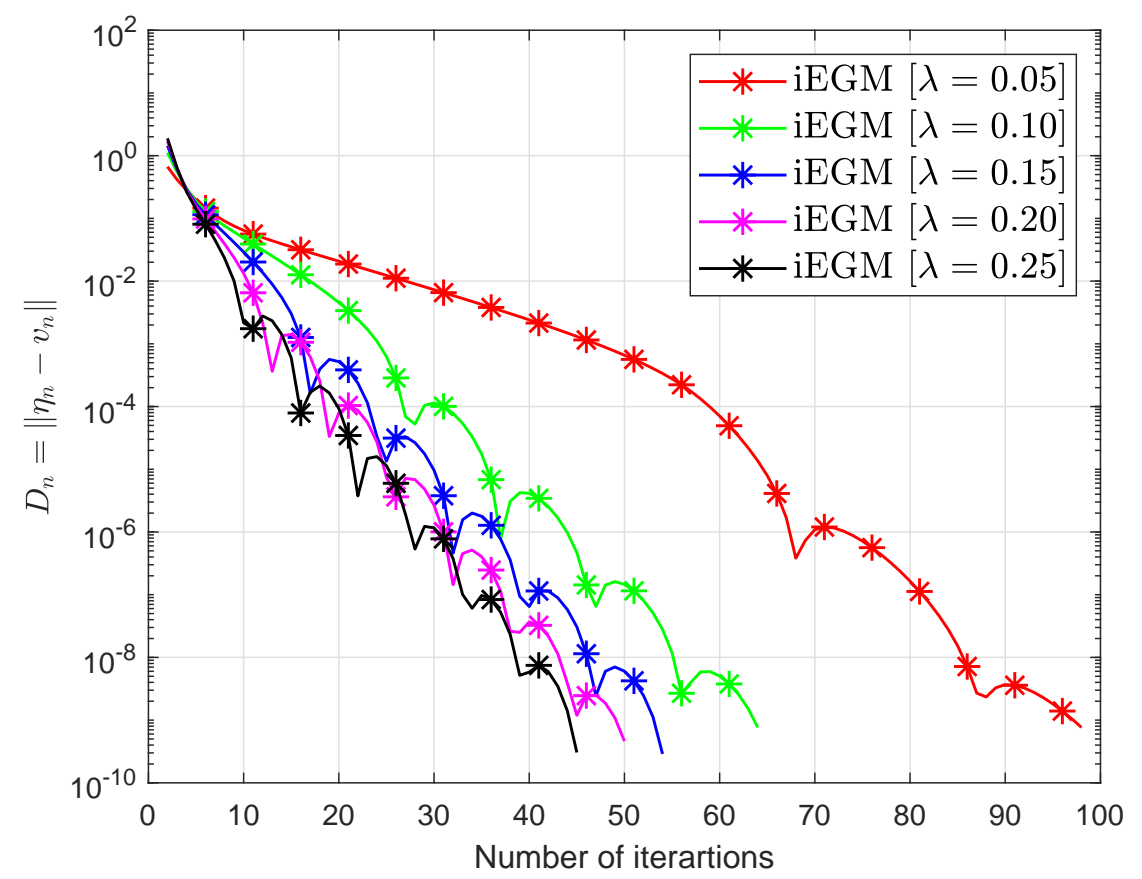

Figure 1. Example 2: numerical behavior of Algorithm 3.1 in [39] by choosing different values of $\lambda$.

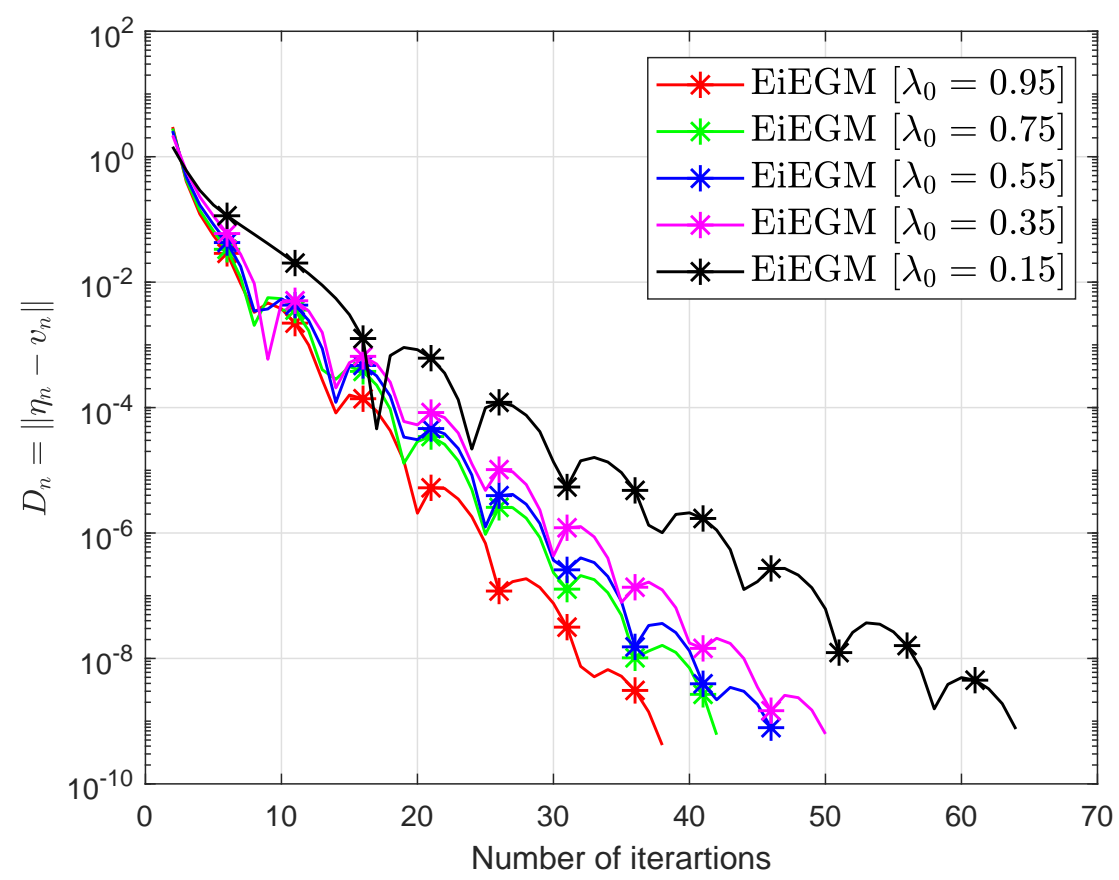

Figure 2. Example 2: numerical behavior of Algorithm 1 by choosing different values of $\lambda_{0}$.

Example 3. Let $f(\breve{p}, \breve{q})=\langle F(\breve{p}), \breve{q}-\breve{p}\rangle$ and $F(\breve{p})=G(\breve{p})+H(\breve{p})$, where

$$
G(\breve{p})=\left(g_{1}(\breve{p}), g_{2}(\breve{p}), \cdots, g_{n}(\breve{p})\right), \quad H(\breve{p})=E \breve{p}+c, c=(-1,-1, \cdots,-1)
$$

and

$$
g_{i}(\breve{p})=\breve{p}_{i-1}^{2}+\breve{p}_{i}^{2}+\breve{p}_{i-1} \breve{p}_{i}+\breve{p}_{i} \breve{p}_{i+1}, \quad i=1,2, \ldots, n, \breve{p}_{0}=\breve{p}_{n+1}=0
$$


The entries of a square matrix E are taken in the following way:

$$
e_{i, j}= \begin{cases}4 & j=i \\ 1 & i-j=1 \\ -2 & i-j=-1 \\ 0 & \text { otherwise, }\end{cases}
$$

where $\mathcal{C}=\left\{\left(u_{1}, \cdots, u_{n}\right) \in \mathcal{R}^{n}: u_{i} \geq 1, i=2, \cdots, n\right\}$. To see the optimum values of the control parameters, some experiments were carried out taking into account the variation of the control parameters $\lambda_{0}$ and the inertial factor $\vartheta$. Figures $3-8$ and Tables 4 and 5 report the numerical results by choosing $u_{-1}=u_{0}=(1, \cdots, 1)$, $\mu=0.33$ and $\epsilon=10^{-6}$.

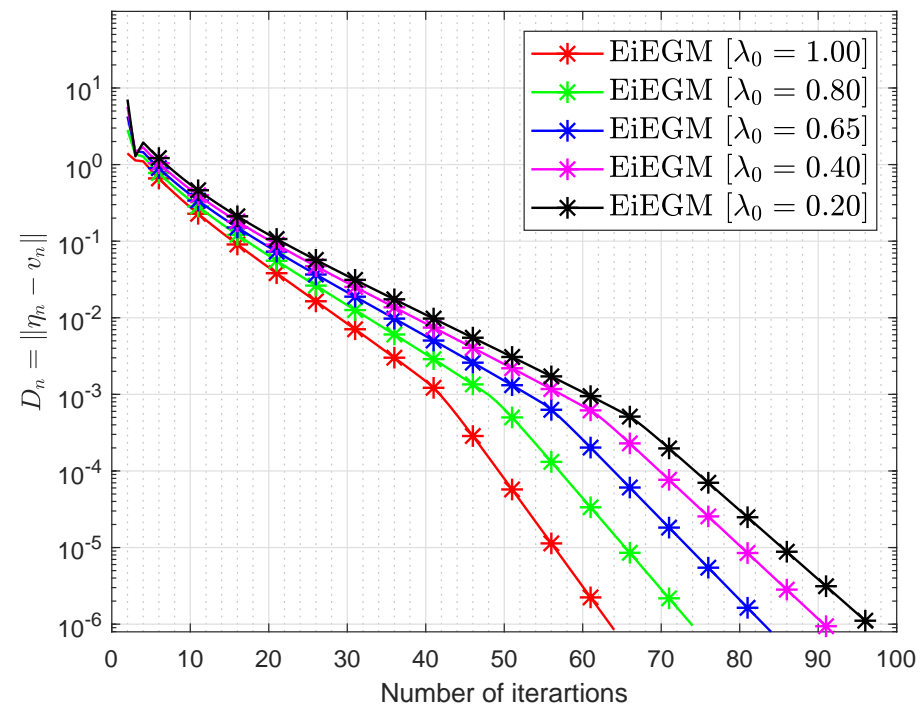

Figure 3. Numerical conduct of Algorithm 1 in $\mathcal{R}^{50}$ by choosing different values of $\lambda_{0}$.

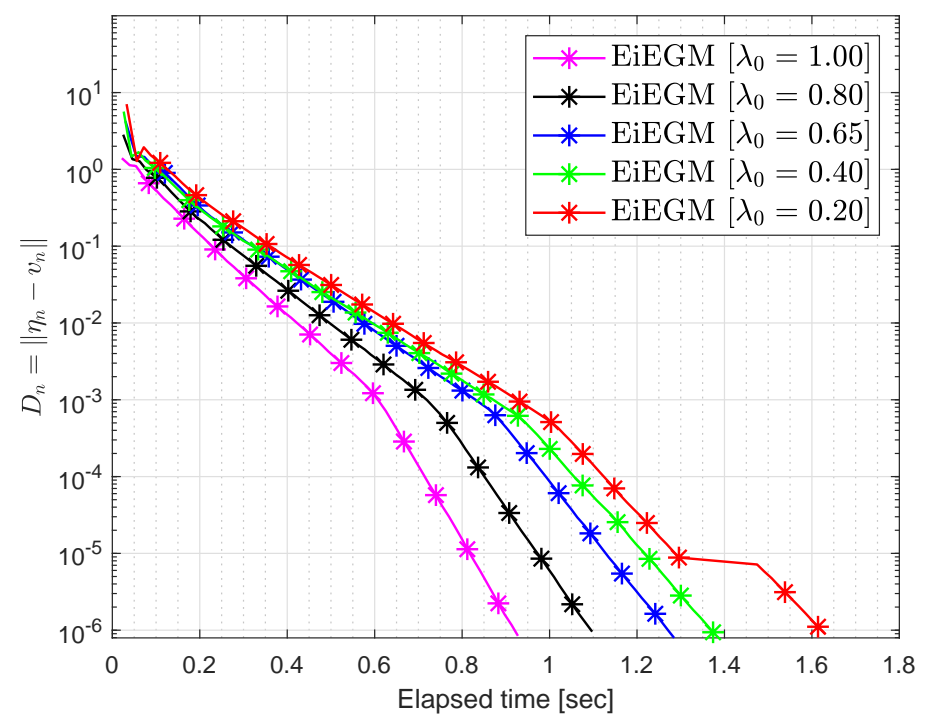

Figure 4. Numerical conduct of Algorithm 1 in $\mathcal{R}^{50}$ by choosing different values of $\lambda_{0}$. 


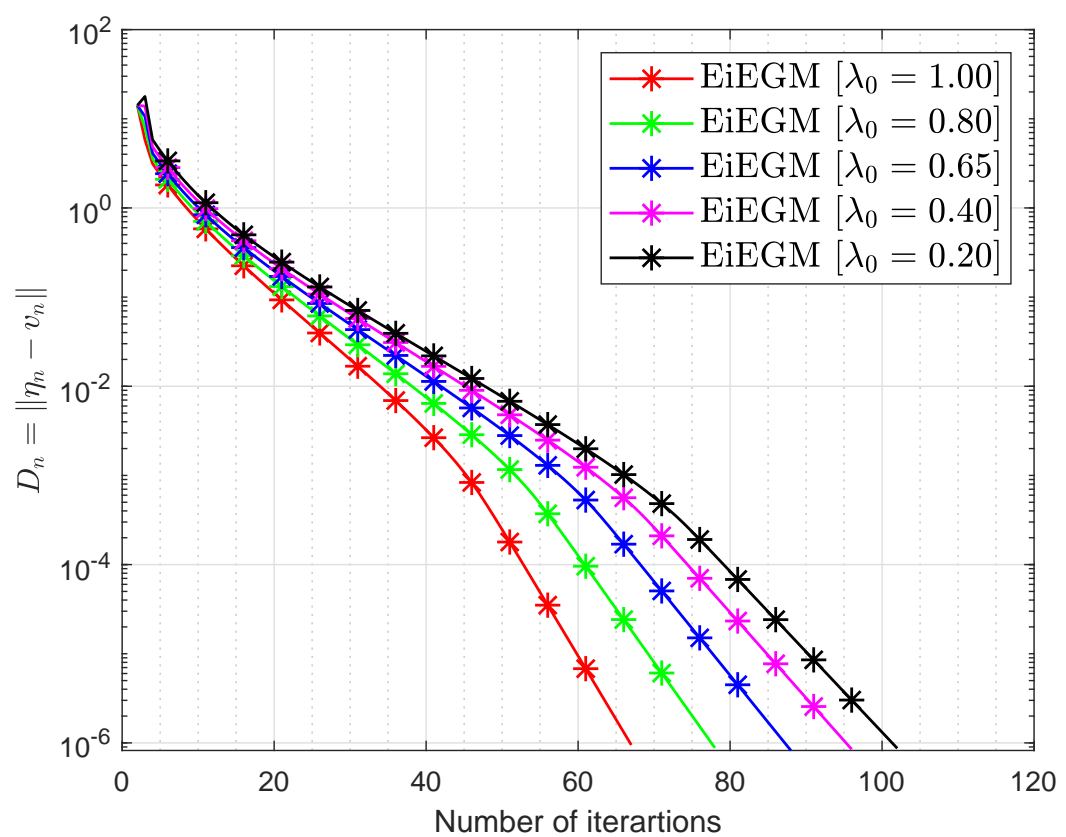

Figure 5. Numerical conduct of Algorithm 1 in $\mathcal{R}^{200}$ by choosing different values of $\lambda_{0}$.

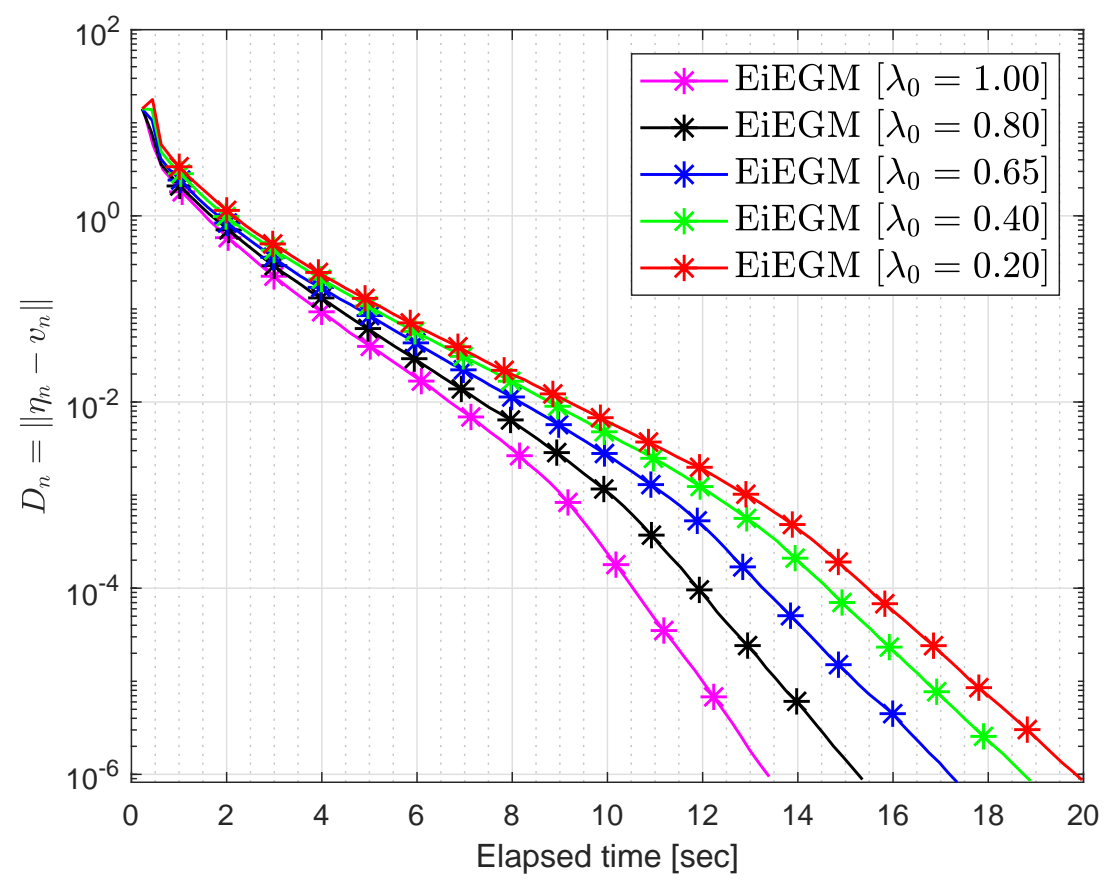

Figure 6. Numerical conduct of Algorithm 1 in $\mathcal{R}^{200}$ by choosing different values of $\lambda_{0}$. 


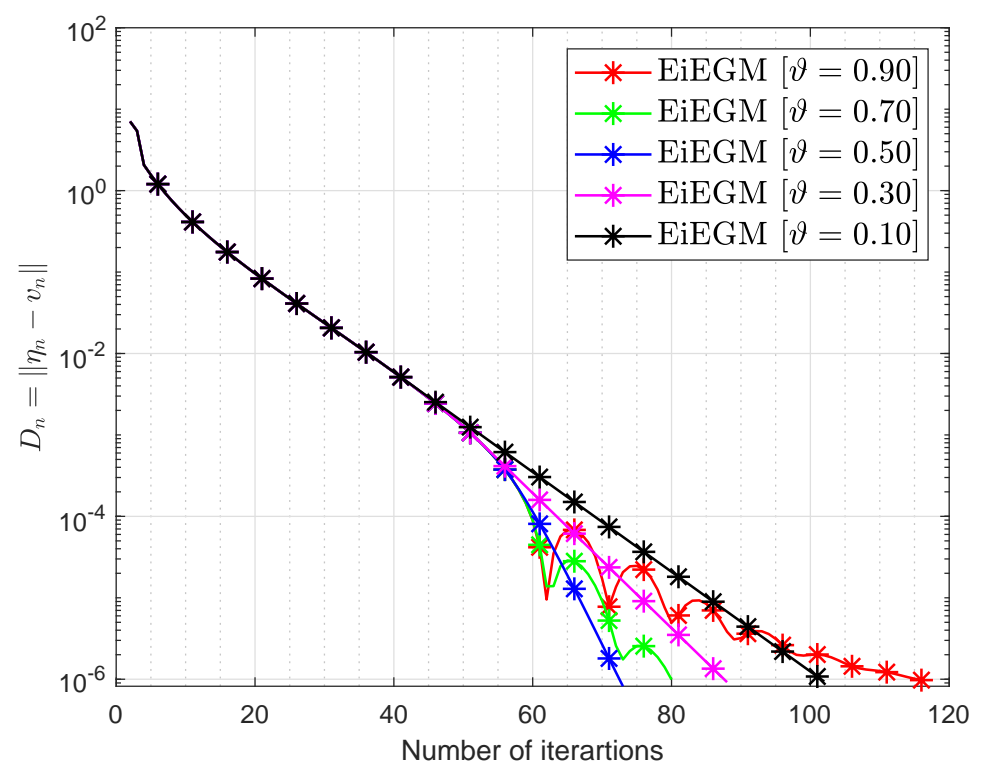

Figure 7. Numerical conduct of Algorithm 1 in $\mathcal{R}^{50}$ by choosing different values of $\vartheta$.

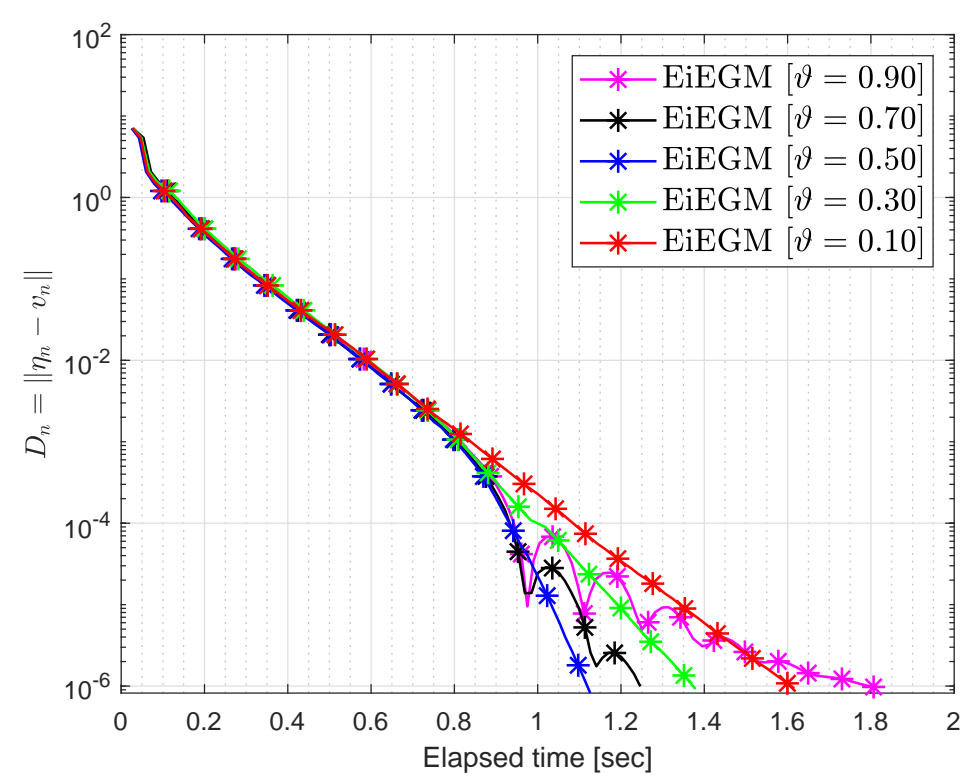

Figure 8. Numerical conduct of Algorithm 1 in $\mathcal{R}^{50}$ by choosing different values of $\vartheta$.

Table 4. Numerical results for Algorithm 1 in $\mathcal{R}^{50}$ by choosing different values of $\lambda_{0}$ and $\vartheta$.

\begin{tabular}{cccccc}
\hline \multicolumn{2}{c}{ EiEGM } & \multicolumn{3}{c}{ EiEGM } \\
\hline$\lambda_{\mathbf{0}}$ & Number of Iterations & CPU Time & $\vartheta$ & Number of Iterations & Execution Time \\
\hline 1.00 & 64 & 0.9276 & 0.90 & 116 & 1.8066 \\
0.80 & 74 & 1.0975 & 0.70 & 80 & 1.2464 \\
0.60 & 84 & 1.2849 & 0.50 & 73 & 1.1262 \\
0.40 & 91 & 1.3740 & 0.30 & 88 & 1.3788 \\
0.20 & 97 & 1.6298 & 0.10 & 102 & 1.6159 \\
\hline
\end{tabular}


Table 5. Numerical results for Algorithm 1 in $\mathcal{R}^{200}$ by choosing different values of $\lambda_{0}$ and $\vartheta$.

\begin{tabular}{cccccc}
\hline & \multicolumn{2}{c}{ EiEGM } & & \multicolumn{2}{c}{ EiEGM } \\
\hline$\lambda_{\mathbf{0}}$ & Number of Iterations & CPU Time & $\boldsymbol{\vartheta}$ & Number of Iterations & Execution Time \\
\hline 1.00 & 67 & 13.3967 & 0.90 & 105 & 20.6972 \\
0.80 & 78 & 15.3566 & 0.70 & 80 & 15.5770 \\
0.60 & 88 & 17.3471 & 0.50 & 80 & 15.4838 \\
0.40 & 96 & 18.8894 & 0.30 & 94 & 19.4532 \\
0.20 & 102 & 19.9705 & 0.10 & 108 & 21.9745 \\
\hline
\end{tabular}

Example 4. Suppose that $\mathcal{H}=L^{2}([0,1])$ is a Hilbert space with an inner product $\langle u, v\rangle=\int_{0}^{1} u(t) v(t) d t, \forall u, v \in \mathcal{H}$ and the induced norm

$$
\|u\|=\sqrt{\int_{0}^{1}|u(t)|^{2} d t}
$$

Assume that $\mathcal{C}:=\left\{u \in L^{2}([0,1]):\|u\| \leq 1\right\}$ is the unit ball. Let $K: \mathcal{C} \rightarrow \mathcal{H}$ be

$$
K(u)(t)=\int_{0}^{1}(u(t)-H(t, s) f(u(s))) d s+g(t),
$$

where

$$
H(t, s)=\frac{2 t s e^{(t+s)}}{e \sqrt{e^{2}-1}}, \quad f(u)=\cos x, \quad g(t)=\frac{2 t e^{t}}{e \sqrt{e^{2}-1}} .
$$

We can see in [48] that $K$ is monotone and Lipschitz-continuous with a Lipschitz constant of $L=2$. Figures 9 and 10 and Table 6 show the numerical results by choosing different values of $u_{0}$ and $\epsilon=10^{-6}$.

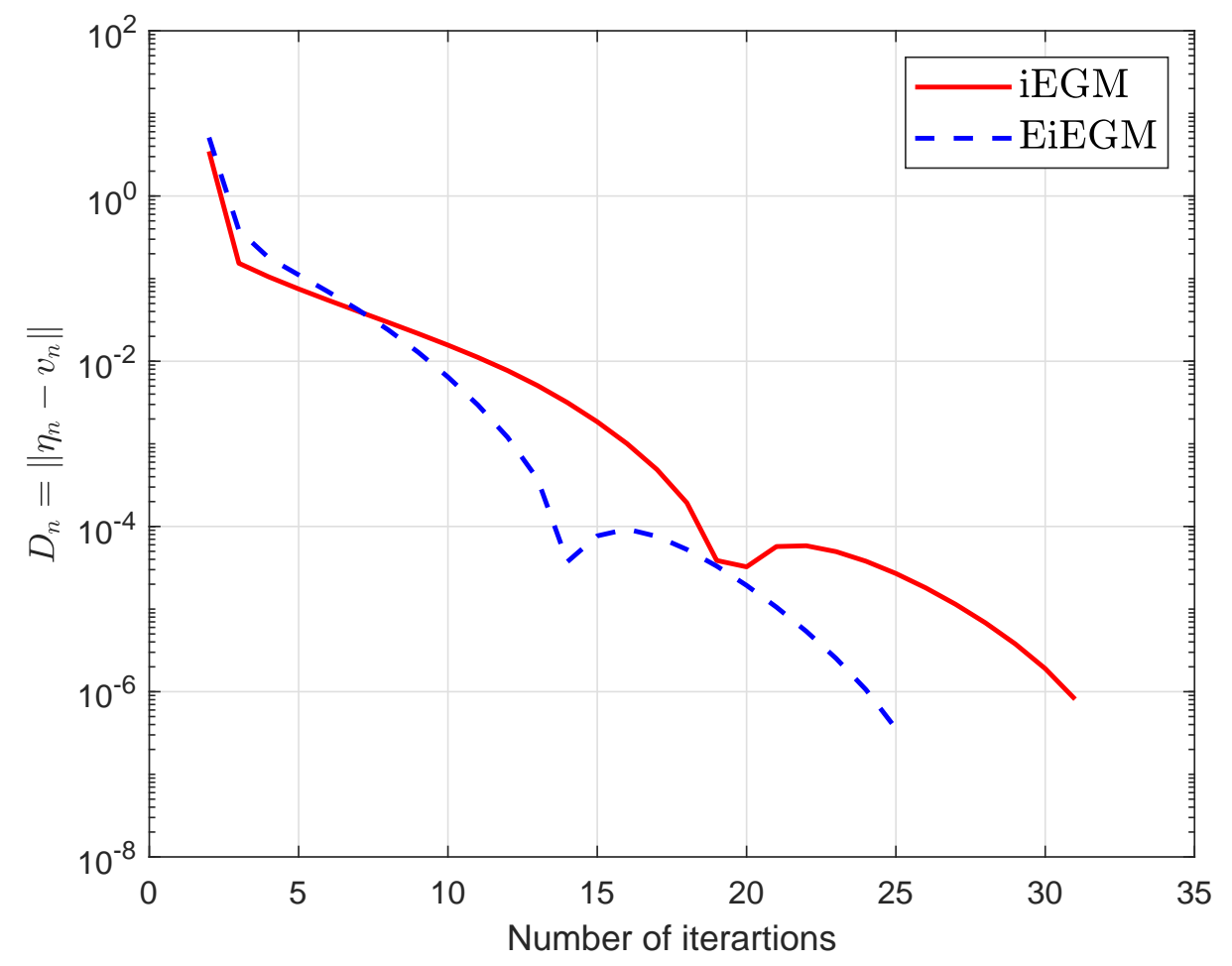

Figure 9. Algorithm 1 comparison with Algorithm 1 in [39] by choosing values of $u_{-1}=u_{0}=1$. 


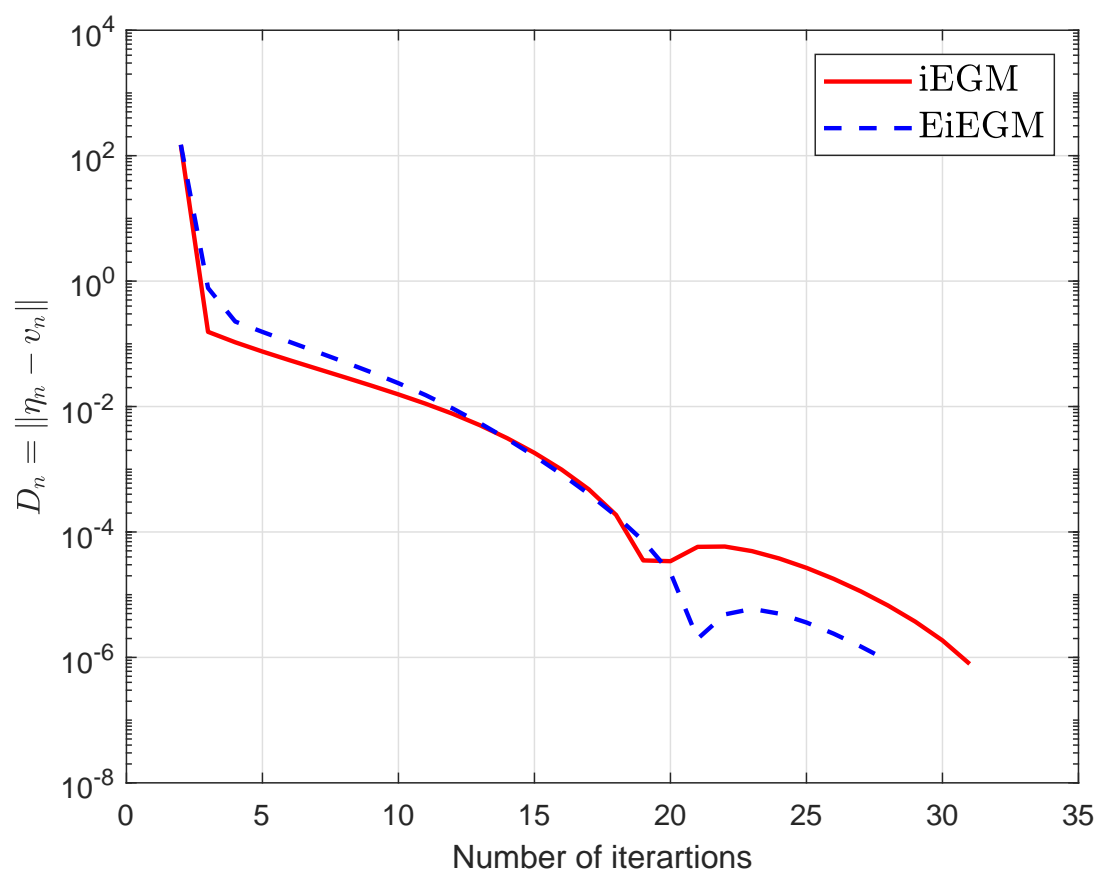

Figure 10. Algorithm 1 comparison with Algorithm 1 in [39] by choosing values of $u_{-1}=u_{0}=t$.

Table 6. Example 4: numerical comparison of Algorithm 1 with Algorithm 1 in [39].

\begin{tabular}{lccccccc}
\hline & & & \multicolumn{3}{c}{ Number of Iterations } & \multicolumn{2}{c}{ Execution Time in Seconds } \\
\hline $\boldsymbol{u}_{-\mathbf{1}}=\boldsymbol{u}_{\mathbf{0}}$ & $\boldsymbol{\vartheta}$ & $\lambda$ & $\lambda_{\mathbf{0}}$ & iEGM & EiEGM & iEGM & EiEGM \\
\hline 1 & 0.50 & 0.20 & 0.50 & 31 & 25 & 0.0158 & 0.0158 \\
$t$ & 0.50 & 0.20 & 0.50 & 31 & 27 & 0.0158 & 0.0158 \\
$2 t^{2}$ & 0.50 & 0.20 & 0.50 & 33 & 30 & 0.0158 & 0.0158 \\
$\sin (t)$ & 0.50 & 0.20 & 0.50 & 37 & 30 & 0.0158 & 0.0158 \\
$\exp (t)$ & 0.50 & 0.20 & 0.50 & 42 & 32 & 0.0158 & 0.0158 \\
\hline
\end{tabular}

Discussion on the Numerical Experiments: We have the following findings about the above-mentioned experiments:

(1) The proposed Algorithm 1 does not depend on the Lipschitz constants, unlike Algorithm 1 in [39]. Algorithm 1 uses a variable stepsize that is updated for each iteration and depends on some of the previous iterations. The key advantage of Algorithm 1 is that it works without prior knowledge of the Lipschitz-type constants $c_{1}$ and $c_{2}$, unlike Algorithm 1 in [39].

(2) Four examples were discussed to compare our proposed method with Algorithm 1 in [39]. In particular, information on Lipschitz constants is missing in Example 3. Due to the missing information of the Lipschitz constants we cannot run Algorithm 1 in [39] because the stepsize $\lambda$ is dependent on Lipschitz constants, i.e., $0<\lambda<\min \left\{\frac{1}{2 c_{1}}, \frac{1}{2 c_{2}}\right\}$. However, we can use the proposed Algorithm 1 to solve Example 3.

(3) It is noted that the selection of the $\vartheta$ value is always important, and precisely the value $\vartheta \in(3,6)$ is better than most other values.

(4) Choosing of the $\lambda_{0}$ value is critical and the proposed algorithm performs better when $\lambda_{0}$ is closer to 1 .

(5) It can also be acknowledged that the efficiency of an algorithms significantly depends on the nature of the problem and tolerance. More time and a considerable number of iterations are needed for large-scale problems. In this case, we could see that a certain stepsize value improves the efficiency of the algorithm and improves the convergence rate. 
(6) Figures 9 and 10 and Table 6 suggest that the choice of initial points and the complexity of the bifunction have an effect on the performance of algorithms in terms of number of iterations and time elapsed.

Author Contributions: Conceptualization, T.B., P.K. and H.u.R.; Writing-Original Draft Preparation, T.B., N.P. and H.u.R.; Writing-Review \& Editing, T.B., N.P., H.u.R., P.K. and W.K.; Methodology, N.P. and H.u.R.; Visualization, T.B. and N.P.; Software, H.u.R.; Funding Acquisition, P.K. and W.K.; Supervision, P.K. and W.K.; Project Administration; P.K. and W.K.; Resources; P.K. and W.K. All authors have read and agreed to the published version of this manuscript.

Funding: This research work was financially supported by King Mongkut's University of Technology Thonburi through the "KMUTT 55th Anniversary Commemorative Fund". Moreover, this project was supported by the Theoretical and Computational Science (TaCS) Center under the Computational and Applied Science for Smart research Innovation research Cluster (CLASSIC), Faculty of Science, KMUTT. In particular, Nuttapol Pakkaranang was supported by the Thailand Research Fund (TRF) through the Royal Golden Jubilee Ph.D. (RGJ-PHD) Program [Grant No. PHD/0205/2561]. Habib ur Rehman was supported by the Petchra Pra Jom Doctoral Academic Scholarship for a Ph.D. Program at KMUTT [grant number 39/2560]. Wiyada Kumam was financially supported by the Rajamangala University of Technology Thanyaburi (RMUTTT) [grant No. NSF62D0604].

Acknowledgments: We are very grateful to the editor and the anonymous referees for their valuable and useful comments, which helped in improving the quality of this work. The second author would like to thank the "Thailand Research Fund (TRF) through the Royal Golden Jubilee Ph.D. (RGJ-PHD) Program (Grant No. PHD/0205/2561)". The third author would like to thank the "Petchra Pra Jom Klao PhD Research Scholarship from the King Mongkut's University of Technology Thonburi".

Conflicts of Interest: The authors declare no conflict of interest.

\section{References}

1. Blum, E. From optimization and variational inequalities to equilibrium problems. Math. Stud. 1994, 63, $123-145$.

2. Bianchi, M.; Schaible, S. Generalized monotone bifunctions and equilibrium problems. J. Optim. Theory Appl. 1996, 90, 31-43. [CrossRef]

3. Fan, K. A Minimax Inequality and Applications, Inequalities III; Shisha, O., Ed.; Academic Press: New York, NY, USA, 1972.

4. Facchinei, F.; Pang, J.S. Finite-Dimensional Variational Inequalities and Complementarity Problems; Springer Science \& Business Media: Berlin, Germany, 2007.

5. Konnov, I. Equilibrium Models and Variational Inequalities; Elsevier: Amsterdam, The Netherlands, 2007; Volume 210.

6. Muu, L.D.; Oettli, W. Convergence of an adaptive penalty scheme for finding constrained equilibria. Nonlinear Anal. Theory Methods Appl. 1992, 18, 1159-1166. [CrossRef]

7. Quoc Tran, D.; Le Dung, M.N.V.H. Extragradient algorithms extended to equilibrium problems. Optimization 2008, 57, 749-776. [CrossRef]

8. Ur Rehman, H.; Kumam, P.; Cho, Y.J.; Yordsorn, P. Weak convergence of explicit extragradient algorithms for solving equilibirum problems. J. Inequalities Appl. 2019, 2019. [CrossRef]

9. Quoc, T.D.; Anh, P.N.; Muu, L.D. Dual extragradient algorithms extended to equilibrium problems. J. Glob. Optim. 2011, 52, 139-159. [CrossRef]

10. Lyashko, S.I.; Semenov, V.V. A new two-step proximal algorithm of solving the problem of equilibrium programming. In Optimization and Its Applications in Control and Data Sciences; Springer International Publishing: Berlin/Heidelberg, Germany, 2016; pp. 315-325. [CrossRef]

11. Takahashi, S.; Takahashi, W. Viscosity approximation methods for equilibrium problems and fixed point problems in Hilbert spaces. J. Math. Anal. Appl. 2007, 331, 506-515. [CrossRef]

12. Ur Rehman, H.; Kumam, P.; Je Cho, Y.; Suleiman, Y.I.; Kumam, W. Modified Popov's explicit iterative algorithms for solving pseudomonotone equilibrium problems. Optim. Methods Softw. 2020, 1-32.

13. Anh, P.N.; Hai, T.N.; Tuan, P.M. On ergodic algorithms for equilibrium problems. J. Glob. Optim. 2015, 64, 179-195. [CrossRef]

14. Hieu, D.V.; Quy, P.K.; Vy, L.V. Explicit iterative algorithms for solving equilibrium problems. Calcolo 2019, 56. [CrossRef]

15. Hieu, D.V. New extragradient method for a class of equilibrium problems in Hilbert spaces. Appl. Anal. 2017, 97, 811-824. [CrossRef] 
16. Ur Rehman, H.; Kumam, P.; Abubakar, A.B.; Cho, Y.J. The extragradient algorithm with inertial effects extended to equilibrium problems. Comput. Appl. Math. 2020, 39. [CrossRef]

17. Santos, P.; Scheimberg, S. An inexact subgradient algorithm for equilibrium problems. Comput. Appl. Math. 2011, 30, 91-107.

18. Ur Rehman, H.; Kumam, P.; Kumam, W.; Shutaywi, M.; Jirakitpuwapat, W. The inertial sub-gradient extra-gradient method for a class of pseudo-monotone equilibrium problems. Symmetry 2020, 12, 463. [CrossRef]

19. Hieu, D.V. Halpern subgradient extragradient method extended to equilibrium problems. Rev. Real Acad. Cienc. Exactas Físicas y Nat. Ser. A Matemáticas 2016, 111, 823-840. [CrossRef]

20. Anh, P.N.; An, L.T.H. The subgradient extragradient method extended to equilibrium problems. Optimization 2012, 64, 225-248. [CrossRef]

21. Ur Rehman, H.; Kumam, P.; Argyros, I.K.; Deebani, W.; Kumam, W. Inertial extra-gradient method for solving a family of strongly pseudomonotone equilibrium problems in real Hilbert spaces with application in variational inequality problem. Symmetry 2020, 12, 503. [CrossRef]

22. Muu, L.D.; Quoc, T.D. Regularization algorithms for solving monotone Ky Fan inequalities with application to a Nash-Cournot equilibrium model. J. Optim. Theory Appl. 2009, 142, 185-204. [CrossRef]

23. Ur Rehman, H.; Kumam, P.; Argyros, I.K.; Alreshidi, N.A.; Kumam, W.; Jirakitpuwapat, W. A self-adaptive extra-gradient methods for a family of pseudomonotone equilibrium programming with application in different classes of variational inequality problems. Symmetry 2020, 12, 523. [CrossRef]

24. Ur Rehman, H.; Kumam, P.; Argyros, I.K.; Shutaywi, M.; Shah, Z. Optimization based methods for solving the equilibrium problems with applications in variational inequality problems and solution of Nash equilibrium models. Mathematics 2020, 8, 822. [CrossRef]

25. Ur Rehman, H.; Kumam, P.; Shutaywi, M.; Alreshidi, N.A.; Kumam, W. Inertial optimization based two-step methods for solving equilibrium problems with applications in variational inequality problems and growth control equilibrium models. Energies 2020, 13, 3292. [CrossRef]

26. Gibali, A.; Hieu, D.V. A new inertial double-projection method for solving variational inequalities. J. Fixed Point Theory Appl. 2019, 21. [CrossRef]

27. Thong, D.V.; Hieu, D.V. Modified subgradient extragradient method for variational inequality problems. Numer. Algorithms 2017, 79, 597-610. [CrossRef]

28. Thong, D.V.; Hieu, D.V. Inertial extragradient algorithms for strongly pseudomonotone variational inequalities. J. Comput. Appl. Math. 2018, 341, 80-98. [CrossRef]

29. Censor, Y.; Gibali, A.; Reich, S. Extensions of Korpelevich's extragradient method for the variational inequality problem in Euclidean space. Optimization 2012, 61, 1119-1132. [CrossRef]

30. Yordsorn, P.; Kumam, P.; Rehman, H.u. Modified two-step extragradient method for solving the pseudomonotone equilibrium programming in a real Hilbert space. Carpathian J. Math. 2020, 36, 313-330.

31. Hammad, H.A.; ur Rehman, H.; la Sen, M.D. Advanced algorithms and common solutions to variational inequalities. Symmetry 2020, 12, 1198. [CrossRef]

32. Gibali, A. A new non-Lipschitzian projection method for solving variational inequalities in Euclidean spaces. J. Nonlinear Anal. Optim. Theory Appl. 2015, 6, 41-51.

33. Dong, Q.L.; Jiang, D.; Gibali, A. A modified subgradient extragradient method for solving the variational inequality problem. Numer. Algorithms 2018, 79, 927-940. [CrossRef]

34. Abubakar, J.; Kumam, P.; ur Rehman, H.; Ibrahim, A.H. Inertial iterative schemes with variable step sizes for variational inequality problem involving pseudomonotone operator. Mathematics 2020, 8, 609. [CrossRef]

35. Abubakar, J.; Sombut, K.; ur Rehman, H.; Ibrahim, A.H. An accelerated subgradient extragradient algorithm for strongly pseudomonotone variational inequality problems. Thai J. Math. 2019, 18.

36. Flåm, S.D.; Antipin, A.S. Equilibrium programming using proximal-like algorithms. Math. Program. 1996, 78, 29-41. [CrossRef]

37. Korpelevich, G. The extragradient method for finding saddle points and other problems. Matecon 1976, 12, 747-756.

38. Yang, J.; Liu, H.; Liu, Z. Modified subgradient extragradient algorithms for solving monotone variational inequalities. Optimization 2018, 67, 2247-2258. [CrossRef]

39. Vinh, N.T.; Muu, L.D. Inertial extragradient algorithms for solving equilibrium problems. Acta Math. Vietnam. 2019, 44, 639-663. [CrossRef] 
40. Censor, Y.; Gibali, A.; Reich, S. The subgradient extragradient method for solving variational inequalities in Hilbert space. J. Optim. Theory Appl. 2010, 148, 318-335. [CrossRef] [PubMed]

41. Kreyszig, E. Introductory Functional Analysis with Applications, 1st ed.; Wiley: New York, NY, USA, 1989.

42. Tiel, J.V. Convex Analysis: An Introductory Text, 1st ed.; Wiley: New York, NY, USA, 1984.

43. Opial, Z. Weak convergence of the sequence of successive approximations for nonexpansive mappings. Bull. Am. Math. Soc. 1967, 73, 591-598. [CrossRef]

44. Tan, K.; Xu, H. Approximating Fixed Points of Nonexpansive Mappings by the Ishikawa Iteration Process. J. Math. Anal. Appl. 1993, 178, 301-308. [CrossRef]

45. Browder, F.; Petryshyn, W. Construction of fixed points of nonlinear mappings in Hilbert space. J. Math. Anal. Appl. 1967, 20, 197-228. [CrossRef]

46. Ur Rehman, H.; Pakkaranang, N.; Hussain, A.; Wairojjana, N. A modified extra-gradient method for a family of strongly pseudomonotone equilibrium problems in real Hilbert spaces. J. Math. Comput. Sci. 2020, 22, 38-48. [CrossRef]

47. Yordsorn, P.; Kumam, P.; ur Rehman, H.; Ibrahim, A.H. A weak convergence self-adaptive method for solving pseudomonotone equilibrium problems in a real Hilbert space. Mathematics 2020, 8, 1165. [CrossRef]

48. Van Hieu, D.; Anh, P.K.; Muu, L.D. Modified hybrid projection methods for finding common solutions to variational inequality problems. Comput. Optim. Appl. 2017, 66, 75-96. [CrossRef]

(C) 2020 by the authors. Licensee MDPI, Basel, Switzerland. This article is an open access article distributed under the terms and conditions of the Creative Commons Attribution (CC BY) license (http://creativecommons.org/licenses/by/4.0/). 